\title{
SHAPE THEORY AND ASYMPTOTIC MORPHISMS FOR C*-ALGEBRAS
}

\author{
MARIUS DADARLAT
}

\section{Introduction}

In this paper we relate two topological invariants of a separable $\mathrm{C}^{*}$-algebras. The first is the shape invariant first studied by Effros and Kaminker [EK] and then developed further by Blackadar [B]. The second invariant is the isomorphism class of a $\mathrm{C}^{*}$-algebra in the asymptotic homotopy category $\mathcal{A}$ introduced by Connes and Higson $[\mathrm{CH}]$. We prove that two separable $\mathrm{C}^{*}$-algebras are shape equivalent if and only if they determine the same class in the category $\mathcal{A}$ (see Theorem 3.9 ). The connection between these two invariants is established via a strong shape invariant that we introduce here. In particular we show that any homotopy invariant functor on separable $\mathrm{C}^{*}$-algebras that commutes with inductive limits automatically factors through the Connes-Higson category and is thus related to K-theory (see Theorem 3.11 ).

It is known that homotopy is less useful in the study of singular spaces. Many interesting $\mathrm{C}^{*}$-algebras (like those associated with infinite discrete groups, dynamical systems and inductive systems) are meant to be noncommutative substitutes for singular spaces. Therefore it is natural to look for weaker forms of homotopy which are better adapted for the study of $\mathrm{C}^{*}$-algebras, but which are still closely allied to homotopy.

In topology, this point of view led Borsuk to introduce shape theory which has become an important area of geometric topology [MS]. Shape theory for $\mathrm{C}^{*}$-algebras was introduced by Effros and Kaminker in [EK1]. Certain key concepts like homotopy continuity and semiprojectivity were isolated there. In a related development Blackadar introduced the notion of semiprojective map and developed a shape theory for all separable $\mathrm{C}^{*}$-algebras $[\mathrm{B}]$. While formally satisfactory the shape theory of $\mathrm{C}^{*}$-algebras has had only few significant examples for which explicit computations were available [EK2], [DN].

Another generalization of homotopy theory for $\mathrm{C}^{*}$-algebras comes from the work of Connes and Higson [CH] and is based on the notion of asymptotic morphism. Roughly speaking an asymptotic morphism from $A$ to $B$ is a continuous family of maps $\varphi_{t}: A \rightarrow B$ which asymptotically satisfies the axioms for $*$-homomorphisms. The asymptotic morphisms of separable $\mathrm{C}^{*}$-algebras can be composed at the level of homotopy giving rise to a category $\mathcal{A}$ called here the asymptotic homotopy category. This category corresponds to a very flexible notion of homotopy which led to the discovery of a concrete realization of E-theory $[\mathrm{H}],[\mathrm{CH}]$ and to the

This research was partially supported by NSF grant DMS-9303361 
construction of non-periodic excision homology and cohomology theories for $\mathrm{C}^{*}$ algebras $[\mathrm{C}],[\mathrm{D}],[\mathrm{CH} 1],[\mathrm{CK}]$. We have explained in $[\mathrm{D}]$ that the Connes-Higson category may be viewed as a noncommutative generalization of the approaching homotopy category of Quigley [Q]. A point of view promoted by these results is that the asymptotic homotopy category $\mathcal{A}$ may be the "right" homotopy category of separable $\mathrm{C}^{*}$-algebras.

With this point of view in mind, in this paper we give results on the asymptotic homotopy classification of inductive limit $\mathrm{C}^{*}$-algebras. We prove that asymptotic homotopy is intimately related to shape theory. Classifying $\mathrm{C}^{*}$-algebras up to an isomorphism in $\mathcal{A}$ may turn out to be a necessary development in the grandiose project of the classification of (simple) nuclear separable $\mathrm{C}^{*}$-algebras $[\mathrm{E}]$.

Let $\mathcal{C}^{*}$ denote the category of separable $\mathrm{C}^{*}$-algebras and $*$-homomorphisms. Let $\mathrm{Ho}\left(\mathrm{C}^{*}\right)$ the category of separable $\mathrm{C}^{*}$-algebras and homotopy classes of *homomorphisms.

In Section 1 of the paper we introduce a homotopy category $H o\left(i n j-\mathcal{C}^{*}\right)$ for inductive systems of separable $\mathrm{C}^{*}$-algebras. This was inspired by similar constructions in the commutative case $[\mathrm{EH}],[\mathrm{L}]$. The category $H o\left(i n j-\mathcal{C}^{*}\right)$ refines the the category $i n j-H o\left(\mathcal{C}^{*}\right)$ which has been used in [EK1] and [B] to construct shape theories. We prove that any isomorphism in $i n j-H o\left(\mathcal{C}^{*}\right)$ lifts to an isomorphism in $H o\left(\operatorname{inj}-\mathcal{C}^{*}\right)$.

In Section 2 we construct a functor $L: H o\left(i n j-\mathcal{C}^{*}\right) \rightarrow \mathcal{A}$ which can be viewed as a homotopy extension of the usual inductive limit functor. Using this functor we show that shape equivalent $\mathrm{C}^{*}$-algebras are asymptotically homotopic. In particular this implies that the homotopy classes of the connecting maps $A_{n} \rightarrow A_{n+1}$ of an inductive system of separable $\mathrm{C}^{*}$-algebras determine the inductive limit $\mathrm{C}^{*}$-algebra $A=\varliminf A_{n}$ up to an asymptotic homotopy (i.e. isomorphism within the category $\mathcal{A})$.

In Section 3 we introduce the strong shape category $\mathcal{S}$. This is the full subcategory of $\mathrm{Ho}\left(\mathrm{inj}_{-} \mathcal{C}^{*}\right)$ whose objects are inductive systems with all the connecting maps semiprojective. We show that $L$ induces an equivalence between the strong shape category $\mathcal{S}$ and the asymptotic homotopy category $\mathcal{A}$. It results that Etheory of Connes and Higson $([\mathrm{H}],[\mathrm{CH}])$ is isomorphic to the strong shape theory of suspensions of stable $\mathrm{C}^{*}$-algebras. Any right inverse of $L$ defines a strong shape functor $S: \mathcal{A} \rightarrow \mathcal{S} \subset \operatorname{Ho}\left(i n j-\mathcal{C}^{*}\right)$ which lifts the shape functor of [B]. A key feature of the strong shape theory of separable $\mathrm{C}^{*}$-algebras is the existence of the adjoint functor $L$ for the strong shape functor $S$. This relates (strong) shape theory to E-theory and hence to K-theory.

The author thanks the referee for a number of suggestions that improved the exposition and the title of the paper. This paper has been circulated as a preprint under the title "Homotopy theory for inductive systems of $\mathrm{C}^{*}$-algebras and asymptotic morphisms".

In the last part of the introduction we review the construction of the category $\mathcal{A}$ due to Connes and Higson.

Let $A, B$ be two $\mathrm{C}^{*}$-algebras. An asymptotic homomorphism from $A$ to $B$ is a family of maps $\varphi_{t}: A \rightarrow B$, indexed by $t \in T=[1,+\infty)$, subject to the following conditions:

(1) For all $a \in A$ the map $t \rightarrow \varphi_{t}(a)$ from $T$ to $A$ is continuous. 
(2) For all $a, b \in A$ and $\lambda \in \mathbb{C}$ one has

$$
\begin{gathered}
\lim _{t \rightarrow \infty}\left\|\varphi_{t}(a+\lambda b)-\varphi_{t}(a)-\lambda \varphi_{t}(b)\right\|=0 \\
\lim _{t \rightarrow \infty}\left\|\varphi_{t}(a b)-\varphi_{t}(a) \varphi_{t}(b)\right\|=0 \\
\lim _{t \rightarrow \infty}\left\|\varphi_{t}\left(a^{*}\right)-\varphi_{t}(a)^{*}\right\|=0
\end{gathered}
$$

Denote by $C_{b}(T, B)$ the $\mathrm{C}^{*}$-algebra of all continuous bounded functions from $T$ to $B$. Let $C_{0}(T, B)$ be the closed ideal of $C_{b}(T, B)$ consisting of functions vanishing at infinity. The quotient $\mathrm{C}^{*}$-algebra $C_{b}(T, B) / C_{0}(T, B)$ is denoted by $B_{\infty}$. Then one has the following exact sequence

$$
0 \rightarrow C_{0}(T, B) \rightarrow C_{b}(T, B) \stackrel{r}{\rightarrow} B_{\infty} \rightarrow 0 .
$$

Since $\lim \sup _{t}\left\|\varphi_{t}(a)\right\| \leq\|a\|$ (see [CH]), one can identify any asymptotic morphism $\left(\varphi_{t}\right): A \rightarrow B$ with a map $\varphi: A \rightarrow C_{b}(T, B)$ given by $\varphi(a)(t)=\varphi_{t}(a)$ for all $a \in A$ and $t \in T$. In the sequel we are going to use freely both notation $\left(\varphi_{t}\right): A \rightarrow B$ and $\varphi: A \rightarrow C_{b}(T, B)$ for asymptotic morphisms. With any asymptotic morphism $\left(\varphi_{t}\right): A \rightarrow B$ one associates a $*$-homomorphism $\hat{\varphi} \in \operatorname{Hom}\left(A, B_{\infty}\right)$ given by $\hat{\varphi}(a)=$ $r(\varphi(a))$, where $r$ is the quotient map of $C_{b}(T, B)$ onto $B_{\infty}$.

Two asymptotic morphisms $\left(\varphi_{t}\right),\left(\psi_{t}\right): A \rightarrow B$ are equivalent, written, $\left(\varphi_{t}\right) \cong\left(\psi_{t}\right)$ if for any $a \in A$ one has $\lim _{t \rightarrow \infty}\left\|\varphi_{t}(a)-\psi_{t}(a)\right\|=0$. Equivalently $\varphi \cong \psi$ iff $\varphi(a)-\psi(a) \in C_{0}(T, B)$ for all $a \in A$.The correspondence $\varphi \mapsto \hat{\varphi}$ induces a bijection from the equivalence classes of asymptotic morphisms from $A$ to $B$ to $\operatorname{Hom}\left(A, B_{\infty}\right)$. Any map $\varphi: A \rightarrow C_{b}(T, B)$ that lifts a given $\hat{\varphi} \in \operatorname{Hom}\left(A, B_{\infty}\right)$ is automatically an asymptotic morphism. Any two liftings are equivalent. Using the selection theorem of Bartle and Graves one can find a continuous

( in general non-additive, but homogeneous) cross-section for the quotient map $r$. It follows then that any asymptotic morphism is equivalent to an asymptotic morphism given by a continuous map $\varphi: A \rightarrow C_{b}(T, B)$. Suppose that the $\mathrm{C}^{*}$-algebra $A$ is separable and nuclear. Then, by the Choi-Effros theorem, any homomorphism $\hat{\varphi}: A \rightarrow B_{\infty}$ has a linear completely positive lifting $\varphi: A \rightarrow C_{b}(T, B)$. One concludes that any asymptotic morphism from $A$ to $B$ is equivalent to a linear completely positive map $\varphi: A \rightarrow C_{b}(T, B)$ satisfying $\varphi(a b)-\varphi(a) \varphi(b) \in C_{0}(T, B)$.

For a $\mathrm{C}^{*}$-algebra $B$ let $B[0,1]$ denote the $\mathrm{C}^{*}$-algebra $C([0,1], B) \cong B \otimes C([0,1])$. Two asymptotic morphisms $\left(\varphi_{t}\right),\left(\psi_{t}\right): A \rightarrow B$ are said to be homotopy equivalent, written $\left(\varphi_{t}\right) \sim\left(\psi_{t}\right)$, if there is an asymptotic morphism $\left(\Phi_{t}\right): A \rightarrow B[0,1]$ such that the restrictions of $\left(\Phi_{t}\right)$ at 0 and 1 are equal to $\left(\varphi_{t}\right)$ and $\left(\psi_{t}\right)$ respectively. Notice that equivalent asymptotic morphisms are homotopy equivalent.

The homotopy classes of asymptotic morphisms from $A$ to $B$ are denoted by $[[A, B]]$. The homotopy class of an asymptotic morphism $\left(\varphi_{t}\right): A \rightarrow B$ is denoted by $\left[\left[\varphi_{t}\right]\right]$ or $[[\varphi]]$. The notation for the homotopy classes of $*$-homomorphisms from $A$ to $B$ is $[A, B]$. There is a composition law of homotopy classes of asymptotic morphisms. We are going to describe briefly this composition. One starts with arbitrary asymptotic morphisms $\left(\varphi_{t}\right): A \rightarrow B$ and $\left(\psi_{t}\right): B \rightarrow C$. As noticed above, after replacing these asymptotic morphisms by equivalent asymptotic morphisms if necessary, one may assume that the maps $\varphi: A \rightarrow C_{b}(T, B)$ and $\psi: B \rightarrow C_{b}(T, C)$ are continuous. Let $A^{\prime}$ be a dense *-subalgebra of $A$ which is a countable union 
of compacts. It is proven in $[\mathrm{CH}]$ that there is a increasing continuous function $\gamma_{0}: T \rightarrow T$ such that for any increasing continuous function $\gamma: T \rightarrow T$ with $\gamma(t) \geq \gamma_{0}(t)$, the composition $\theta_{t}^{\prime}=\psi_{s(t)} \circ \varphi_{t}$ is an asymptotic morphism from $A^{\prime}$ to $C$. Moreover $\limsup _{t}\left\|\theta_{t}^{\prime}(a)\right\| \leq\|a\|$ for all $a \in A^{\prime}$. Thus the associated map $\theta^{\prime}: A^{\prime} \rightarrow C_{b}(T, C)$ is well defined so that $\hat{\theta}^{\prime}: A^{\prime} \rightarrow C_{\infty}$ is a bounded *homomorphism that extends to a $*$-homomorphism $\hat{\theta}$ on $A$. Let $\left(\theta_{t}\right)$ be any lifting of $\hat{\theta}$. By definition $\left[\left[\psi_{t}\right]\right] \circ\left[\left[\varphi_{t}\right]\right]=\left[\left[\theta_{t}\right]\right]$.

Theorem $([\mathrm{CH}])$ Any extension $\left(\theta_{t}\right)$ of $\left(\phi_{s(t)} \circ \varphi_{t}\right)$ is an asymptotic morphism. The homotopy class of $\left[\left[\theta_{t}\right]\right]$ in $[[A, C]]$ depends only on the homotopy classes of $\left[\left[\varphi_{t}\right]\right] \in[[A, B]]$ and $\left[\left[\psi_{t}\right]\right] \in[[B, C]]$. Moreover the composition of homotopy classes $\left[\left[\psi_{t}\right]\right] \circ\left[\left[\varphi_{t}\right]\right]$ is associative.

This theorem shows that there is a well defined associative composition law

$$
[[A, B]] \times[[B, C]] \rightarrow[[A, C]]
$$

By definition $\mathcal{A}$ is the the category whose objects are all the separable $C^{*}$-algebras and whose morphisms are homotopy classes of asymptotic morphisms.

\section{Homotopy theory for inductive systems of $\mathrm{C}^{*}$-algebras}

Let $\mathcal{C}^{*}$ denote the category of separable $\mathrm{C}^{*}$-algebras and *-homomorphisms. Let $H o\left(\mathcal{C}^{*}\right)$ the category of separable $\mathrm{C}^{*}$-algebras and homotopy classes of *homomorphisms.

Definition 1.1 (The category $i n j-\mathcal{C}^{*}$ ) The objects of $i n j-\mathcal{C}^{*}$ are inductive systems $\left(A_{n}, p_{n+1 n}\right)$ of separable $\mathrm{C}^{*}$-algebras indexed by the natural numbers.

$$
\ldots \rightarrow A_{n} \stackrel{p_{n+1 n}}{\longrightarrow} A_{n+1} \rightarrow \ldots
$$

For $m>n$ set $p_{m n}=p_{m m-1} \ldots p_{n+1 n}$. A morphism of inductive systems $\underline{f}: \underline{A} \rightarrow$ $\underline{B}=\left(B_{n}, q_{n+1 n}\right)$ consists of an increasing map $f: \mathbb{N} \rightarrow \mathbb{N}$ (called the indexing map) and a of sequence of $*$-homomorphisms $f_{n}: A_{n} \rightarrow B_{f(n)}$ such that the following diagram commutes:

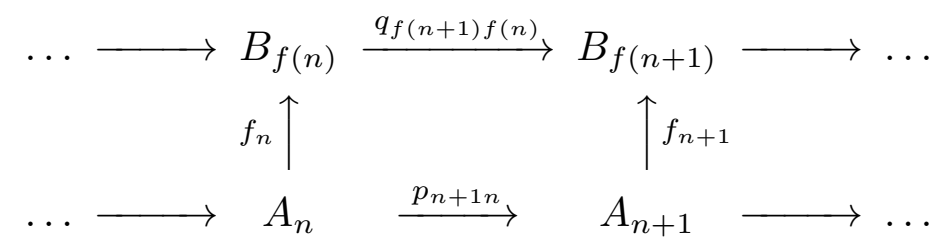

Two morphisms of systems $\underline{f}, \underline{f}^{\prime}: \underline{A} \rightarrow \underline{B}$ are equivalent if for each $n$ there is $m \geq f(n), f^{\prime}(n)$ such that $q_{m f(n)} f_{n}=q_{m f^{\prime}(n)} f_{n}^{\prime}$. The morphisms of $i n j-\mathcal{C}^{*}$ are the equivalence classes of morphisms of systems.

1.2 Consider the inductive limit functor

$$
\stackrel{\lim }{\longrightarrow}: i n j-\mathcal{C}^{*} \rightarrow \mathcal{C}^{*}
$$

This functor takes an inductive system of separable $\mathrm{C}^{*}$-algebras $\underline{A}=\left(A_{n}, p_{n+1 n}\right)$ to its inductive limit $\mathrm{C}^{*}$-algebra $A=\underline{\lim } \underline{A}$. Let $p_{n}$ be the canonical mapping 
of $A_{n}$ into $A$. A morphism $\underline{f}: \underline{A} \rightarrow \underline{B}$ in $i n j-\mathcal{C}^{*}$ induces a $*$-homomorphism $\hat{f}=\underline{\lim } \underline{f}: \underline{\lim } \underline{A} \rightarrow \underline{\lim } \underline{B}=B$ such that $\hat{f} p_{n}=q_{f(n)} f_{n}$.

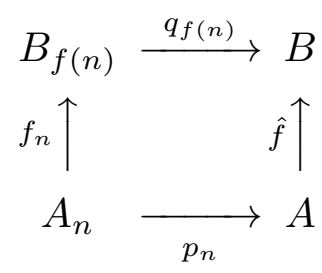

Definition 1.3 (The category $\left.i n j-H o\left(\mathcal{C}^{*}\right)\right)$ The objects of $i n j-H o\left(\mathcal{C}^{*}\right)$ are inductive systems $\underline{A}=\left(A_{n}, p_{n+1 n}\right)$ of separable $\mathrm{C}^{*}$-algebras indexed by the natural numbers. A map of systems $f: \underline{A} \rightarrow \underline{B}=\left(B_{n}, q_{n+1 n}\right)$ consists of an increasing map $f: \mathbb{N} \rightarrow \mathbb{N}$ and a sequence of $*$-homomorphisms $f_{n}: A_{n} \rightarrow B_{f(n)}$ such that the diagram (1) commutes up to a homotopy. That is $q_{f(n+1) f(n)} f_{n}$ is homotopic to $f_{n+1} p_{n+1 n}$ for all $n \in \mathbb{N}$.

Two maps of systems $\underline{f}, \underline{f}^{\prime}: \underline{A} \rightarrow \underline{B}$ are equivalent if for each $n$ there is $m \geq$ $f(n), f^{\prime}(n)$ such that $q_{m f(n)} f_{n}$ is homotopic to $q_{m f^{\prime}(n)} f_{n}^{\prime}$. The morphisms of $i n j-$ $H o\left(\mathcal{C}^{*}\right)$ are the homotopy classes of maps of systems. The set of morphisms from $\underline{A}$ to $\underline{B}$ is denoted by $[\underline{A}, \underline{B}]$. The homotopy class of $\underline{f}: \underline{A} \rightarrow \underline{B}$ is denoted by $[\underline{f}]$.

1.4 Various subcategories of $i n j-H o\left(\mathcal{C}^{*}\right)$ were considered in [EK1] and [B] in connection with shape theories for $\mathrm{C}^{*}$-algebras. One may regard $i n j-H o\left(\mathcal{C}^{*}\right)$ as a homotopy category of $i n j-\mathcal{C}^{*}$. However, this point of view is inadequate for some purposes. For instance the inductive limit functor does not descend to a functor $i n j-H o\left(\mathcal{C}^{*}\right) \rightarrow H o\left(\mathcal{C}^{*}\right)$. There are at least two reasons that prevent this happening. The category $\mathrm{Ho}\left(\mathcal{C}^{*}\right)$ is too rigid and the morphisms of $i n j-H o\left(\mathcal{C}^{*}\right)$ do not carry enough coherent geometrical data. Fortunately Connes and Higson have discovered the category $\mathcal{A}$ of separable $\mathrm{C}^{*}$-algebras and homotopy classes of asymptotic morphisms. This has proven to be the "right" homotopy category of $\mathcal{C}^{*}$ and a flexible substitute of $\mathrm{Ho}\left(\mathcal{C}^{*}\right)$ ( see $\left.[\mathrm{CH}],[\mathrm{CK}],[\mathrm{D}]\right)$.

In analogy with the commutative case $[\mathrm{EH}]$, [L] we introduce here the category $H o\left(i n j-\mathcal{C}^{*}\right)$ which we believe to be the "right" homotopy category of $i n j-\mathcal{C}^{*}$. Indeed, in the next section we construct a functor $L: H o\left(i n j-\mathcal{C}^{*}\right) \rightarrow \mathcal{A}$ such that the following diagram is commutative:

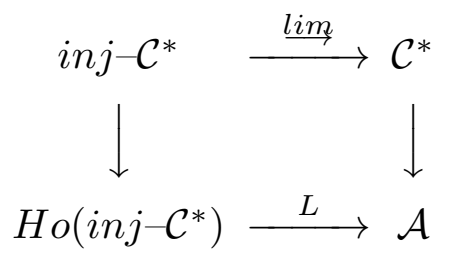

Definition 1.5 (The category $\left.H o\left(i n j-\mathcal{C}^{*}\right)\right)$ The objects of $H o\left(i n j-\mathcal{C}^{*}\right)$ are inductive systems $\left(A_{n}, p_{n+1 n}\right)$ of separable $\mathrm{C}^{*}$-algebras. A strong map of systems $(\underline{f}, \underline{h}): \underline{A} \rightarrow \underline{B}=\left(B_{n}, q_{n+1 n}\right)$ consists of an increasing indexing map $f: \mathbb{N} \rightarrow \mathbb{N}$, of a sequence of $*$-homomorphisms $f_{n}: A_{n} \rightarrow B_{f(n)}$ and of a sequence of $*$ homomorphisms (homotopies) $h_{n}: A_{n} \rightarrow B_{f(n+1)}[0,1], h_{n}=\left(h_{n}^{\tau}\right)_{\tau \in[0,1]}$, such that

$$
\begin{aligned}
& h_{n}^{0}=q_{f(n+1) f(n)} f_{n} \\
& h_{n}^{1}=f_{n+1} p_{n+1 n}
\end{aligned}
$$


Diagramatically we represent $(\underline{f}, \underline{h})$ by

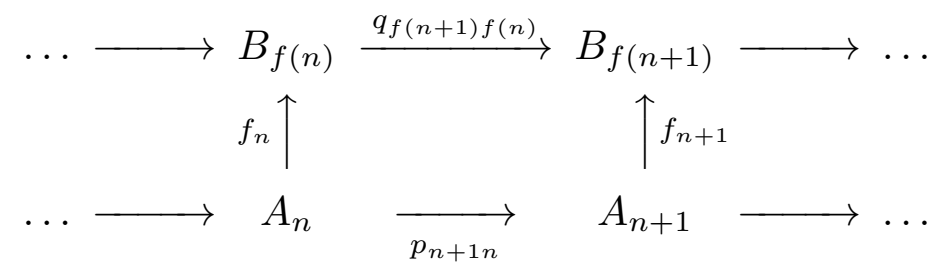

A strong map of systems stores more information than a map in $i n j-H o\left(\mathcal{C}^{*}\right)$ because it keeps track of the homotopies $h_{n}^{\tau}$ that are filling each homotopy commutative square. Note that any morphism $\underline{f}: \underline{A} \rightarrow \underline{B}$ of inductive systems (see 1.1 ) induces a strong map of systems $\underline{A} \rightarrow \underline{B}$ with constant homotopies $h_{n}^{\tau}=f_{n+1} p_{n+1 n}$. Two strong maps of inductive systems $(f, \underline{h}),\left(f^{\prime}, \underline{h}^{\prime}\right): \underline{A} \rightarrow \underline{B}$ are called homotopic if for each $n \in \mathbb{N}$ there is $\nu(n) \geq f(n), f^{\prime}(n)$ and there is a $*$-homomorphism $\nu_{n}: A_{n} \rightarrow B_{\nu(n)}[0,1], \quad \nu_{n}=\left(\nu_{n}^{s}\right)_{s \in[0,1]}$ such that

$$
\begin{aligned}
& \nu_{n}^{0}=q_{\nu(n) f(n)} f_{n} \\
& \nu_{n}^{1}=q_{\nu(n) f^{\prime}(n)} f_{n}^{\prime}
\end{aligned}
$$

Moreover we require the existence of a two-parameter homotopy $\mu_{n}: A_{n} \rightarrow B_{\nu(n+1)}[0,1] \times[0,1], \quad \mu_{n}=\left(\mu_{n}^{s, \tau}\right)_{(s, \tau) \in[0,1] \times[0,1]}$, such that

$$
\begin{aligned}
& \mu_{n}^{s, 0}=q_{\nu(n+1) \nu(n)} \nu_{n}^{s} \\
& \mu_{n}^{s, 1}=\nu_{n+1}^{s} p_{n+1 n} \\
& \mu_{n}^{0, \tau}=q_{\nu(n+1) f(n+1)} h_{n}^{\tau} \\
& \mu_{n}^{1, \tau}=q_{\nu(n+1) f^{\prime}(n+1)} h_{n}^{\prime \tau}
\end{aligned}
$$

Two strong maps of systems are called equivalent if for each $n$ there is $m \geq$ $f(n), f^{\prime}(n)$ such that $q_{m f(n+1)} h_{n}^{\tau}=q_{m f^{\prime}(n+1)} h_{n}^{\prime \tau}$. Note that equivalent strong maps of inductive systems are homotopic. One checks that homotopy is an equivalence relation.

By definition the morphisms of $H o\left(i n j-\mathcal{C}^{*}\right)$ are homotopy classes of strong maps of inductive systems. The set of morphisms from $\underline{A}$ to $\underline{B}$ is denoted by $[[\underline{A}, \underline{B}]]$. The homotopy class of $(f, \underline{h}): \underline{A} \rightarrow \underline{B}$ is denoted by $[[(f, \underline{h})]]$.

The composition of morphisms in $H o\left(i n j-\mathcal{C}^{*}\right)$ is discussed in $1.6-1.9$.

1.6 An increasing map $\alpha: \mathbb{N} \rightarrow \mathbb{N}$ induces a map $(\underline{f}, \underline{h}) \rightarrow\left(\underline{f}_{\alpha}, \underline{h}_{\alpha}\right)$. The strong map $\left(\underline{f}_{\alpha}, \underline{h}_{\alpha}\right)$ is described by the diagram

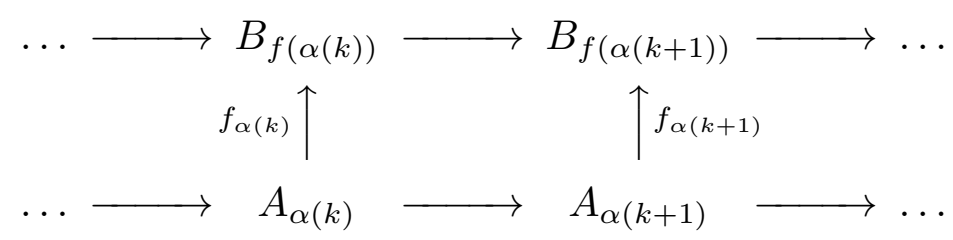

where $h_{\alpha, k}^{\tau}$ is a suitable juxtaposition of homotopies involving $\left\{h_{i}^{\tau}\right\}$ for $\alpha(k) \leq i \leq \alpha(k+1)-1$. This is shown in the diagram

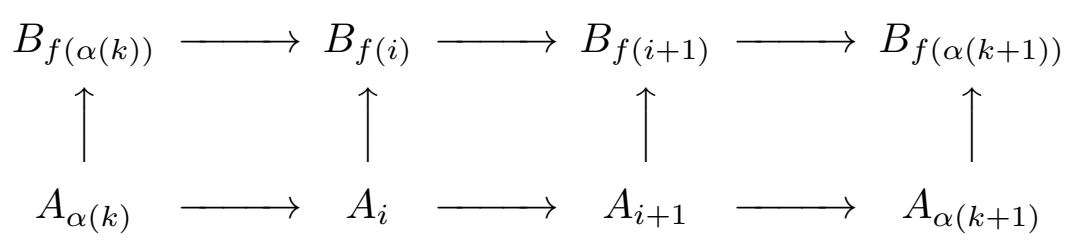


More precisely we set

$$
h_{\alpha, k}^{\tau}=q_{f(\alpha(k+1)) f(i+1)} h_{i}^{m \tau-i} p_{i \alpha(k)}
$$

where $m=\alpha(k+1)-\alpha(k)$ and $0 \leq i / m \leq \tau \leq(i+1) / m \leq 1$.

1.7 The composition $\left(f^{\prime \prime}, \underline{h}^{\prime \prime}\right)=\left(\underline{f}^{\prime}, \underline{h}^{\prime}\right) \circ(\underline{f}, \underline{h})$ of two strong maps of systems $(\underline{f}, \underline{h}): \underline{A} \rightarrow \underline{B}$ and $\left(\underline{f}^{\prime}, \underline{h}^{\prime}\right): \underline{B} \rightarrow \underline{C}=\left(C_{n}, r_{n+1 n}\right)$ is the strong map of inductive systems consisting of an increasing map $f^{\prime \prime}: \mathbb{N} \rightarrow \mathbb{N}, f^{\prime \prime}(n)=f^{\prime} f(n)$, of a sequence of *-homomorphisms $f_{n}^{\prime \prime}: A_{n} \rightarrow C_{f^{\prime} f(n)}, f_{n}^{\prime \prime}=f_{f(n)}^{\prime} f_{n}$ and of homotopies $h_{n}^{\prime \prime}$ : $A_{n} \rightarrow C_{f^{\prime} f(n+1)}[0,1]$ given by

$$
h_{n}^{\prime \prime \tau}=\left\{\begin{array}{lll}
h_{f, n}^{\prime 2 \tau} f_{n}, & \text { if } & 0 \leq \tau \leq 1 / 2 \\
f_{f(n+1)}^{\prime} h_{n}^{2 \tau-1}, & \text { if } & 1 / 2 \leq \tau \leq 1
\end{array}\right.
$$

In other words, one passes from $\left(\underline{f}^{\prime}, \underline{h}^{\prime}\right)$ to $\left(\underline{f}_{f}^{\prime}, \underline{h}_{f}^{\prime}\right)$ ( as in 1.6 with $\alpha=f=$ the indexing map of $(\underline{f}, \underline{h}))$ and then the diagrams representing $\left(\underline{f}_{f}^{\prime}, \underline{h}_{f}^{\prime}\right)$ and $(\underline{f}, \underline{h})$ are juxtaposed.

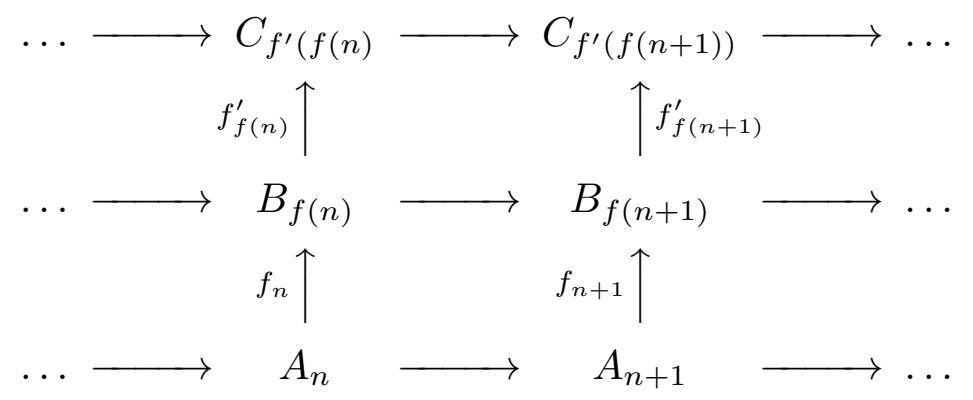

1.8 The composition of strong maps of systems passes to homotopy classes. To prove this we begin by reexamining the definition of homotopy given in 1.5. For an inductive system $\underline{B}=\left(B_{n}, q_{n+1 n}\right)$ let $\underline{B}[0,1]$ denote the inductive system

$\left(B_{n}[0,1], q_{n+1 n} \otimes 1_{C([0,1])}\right)$. The evaluation maps $e_{n}^{s}: B_{n}[0,1] \rightarrow B_{n}$, at $s \in[0,1]$ give rise to an obvious strong map of systems $\underline{e}^{s}: \underline{B}[0,1] \rightarrow \underline{B}$ with constant homotopies. The sequences $\left(\nu_{n}\right)$ and $\left(\mu_{n}\right)$ of Definition 1.5 give rise to a strong map of systems

$(\underline{\nu}, \underline{\mu}): \underline{A} \rightarrow \underline{B}[0,1]$. This leads to the following

Remark Two strong maps of inductive systems $\left(\underline{f}^{\prime}, \underline{h}^{\prime}\right),\left(\underline{f}^{\prime \prime}, \underline{h}^{\prime \prime}\right): \underline{B} \rightarrow \underline{C}$ are homotopic if and only if there is a strong map of systems $(\underline{\nu}, \bar{\mu}): \underline{B} \rightarrow \underline{C}[0,1]$ such that $\underline{e^{0}}(\underline{\nu}, \underline{\mu})$ is equivalent to $\left(\underline{f}^{\prime}, \underline{h}^{\prime}\right)$ and $\underline{e^{1}}(\underline{\nu}, \underline{\mu})$ is equivalent to $\left(\underline{f}^{\prime \prime}, \underline{h}^{\prime \prime}\right)$ (see the last part of 1.5). If $\alpha: \mathbb{N} \rightarrow \mathbb{N}$ is an increasing function then $\left(\underline{\nu}_{\alpha}, \underline{\mu}_{\alpha}\right)$ is a homotopy from $\left(\underline{f}_{\alpha}^{\prime}, \underline{h}_{\alpha}^{\prime}\right)$ to $\left(\underline{f}_{\alpha}^{\prime \prime}, \underline{h}_{\alpha}^{\prime \prime}\right)$.

Proposition 1.9 The homotopy class of the composition $\left(f^{\prime}, \underline{h}^{\prime}\right) \circ(f, \underline{h})$ depends only upon the homotopy classes of $\left(\underline{f}^{\prime}, \underline{h}^{\prime}\right)$ and $(\underline{f}, \underline{h})$. Thus there is a well defined associative composition of morphisms

$$
[[\underline{A}, \underline{B}]] \times[[\underline{B}, \underline{C}]] \rightarrow[[\underline{A}, \underline{C}]]
$$

that makes $H o\left(i n j-\mathcal{C}^{*}\right)$ a category. 
Proof (sketch) Using the above Remark one forms compositions of homotopies. This reduces the statement to showing that compositions of equivalent strong maps with a strong map on any side are homotopic. A proof of this requires only standard arguments and is omitted.

1.10 There is a useful procedure for reindexing strong maps of systems. Given an increasing map $\alpha: \mathbb{N} \rightarrow \mathbb{N}$, let $\underline{i}_{\alpha}$ denote the strong map of systems defined by the commutative diagram

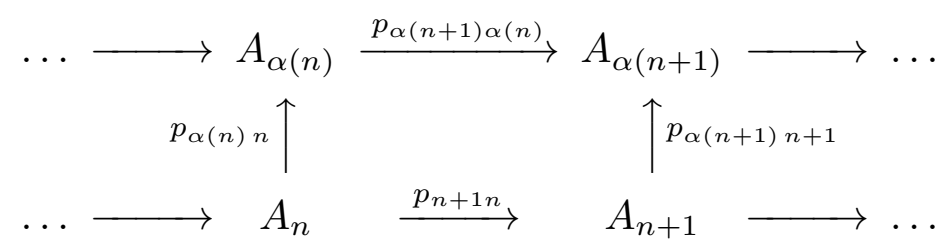

The following Lemma is obvious.

Lemma $\left[\left[\underline{i}_{\alpha}\right]\right]: \underline{A} \rightarrow \underline{A}$ is equal to the identity morphism of $\underline{A}$ in $H o\left(i n j-\mathcal{C}^{*}\right)$.

Lemma 1.11 Let $(\underline{f}, \underline{h}): \underline{A} \rightarrow \underline{B}$ be a strong map of inductive systems and let $\alpha, \beta: \mathbb{N} \rightarrow \mathbb{N}$ be increasing maps. Then $\underline{i}_{\beta} \circ\left((\underline{f}, \underline{h}) \circ \underline{i}_{\alpha}\right)$ is homotopic to the $(\underline{f}, \underline{h})$.

Proof. This follows from Proposition 1.9 and Lemma 1.10.

Lemma 1.12 Let $\underline{A}=\left(A_{n}, p_{n+1 n}\right)$ and $\underline{B}=\left(A_{n}, q_{n+1 n}\right)$ be inductive systems of separable $\mathrm{C}^{*}$-algebras. Assume that $p_{n+1 n}$ is homotopic to $q_{n+1 n}$ for each $n$. Then $\underline{A}$ is isomorphic to $\underline{B}$ in $H o\left(i n j-\mathcal{C}^{*}\right)$.

Proof Let $h_{n}: A_{n} \rightarrow B_{n+1}[0,1]$ be a homotopy from $p_{n+1 n}$ to $q_{n+1 n}$. Then $x=\left(i d_{A_{n}}, h_{n}\right): \underline{A} \rightarrow \underline{B}$ is a strong map of systems. Let $y=\left(i d_{A_{n}}, h_{n}^{\prime}\right): \underline{B} \rightarrow \underline{A}$ be a strong map of systems with $h_{n}^{\prime \tau}=h_{n}^{1-\tau}$. Let $(\underline{\nu}, \underline{\mu}): \underline{B} \rightarrow \underline{A}[0,1]$ be given by the sequences of $*$-homomorphisms: $\nu_{n}^{s}=i d_{A_{n}}$ and $\mu_{n}: A_{n} \rightarrow A_{n+1}[0,1] \times[0,1]$,

$$
\mu_{n}^{s, \tau}=\left\{\begin{array}{lll}
h_{n}^{2 s \tau}, & \text { if } & 0 \leq \tau \leq 1 / 2 \\
h_{n}^{(2-2 \tau) s}, & \text { if } & 1 / 2 \leq \tau \leq 1
\end{array}\right.
$$

Then $(\underline{\nu}, \underline{\mu})$ is a homotopy from the identity of $\underline{B}$ to $x y$. By symmetry $y x$ is homotopic to the identity of $\underline{A}$.

1.13 It follows from the construction of the category $\mathrm{Ho}\left(i n j-\mathcal{C}^{*}\right)$ that there is a canonical functor

$$
F: H o\left(i n j-\mathcal{C}^{*}\right) \rightarrow i n j-H o\left(\mathcal{C}^{*}\right) .
$$

The functor $F$ acts as identity on objects and sends the homotopy class of $(f, \underline{h})$ to the homotopy class of $\underline{f}$. That is $F[[(\underline{f}, \underline{h})]]=[\underline{f}]$. It is obvious that for inductive systems $\underline{A}$ and $\underline{B}, F$ induces a surjective map

$$
[[\underline{A}, \underline{B}]] \rightarrow[\underline{A}, \underline{B}] .
$$

In general this map is not injective even for commutative $\mathrm{C}^{*}$-algebras [MS].

However one has the following noncommutative version of Theorem 5.4.1 in [EH].

Theorem 1.14 Any isomorphism in $i n j-H o\left(\mathcal{C}^{*}\right)$ lifts to an isomorphism in $H o\left(\operatorname{inj}-\mathcal{C}^{*}\right)$.

Proof Let $\underline{f}: \underline{A} \rightarrow \underline{B}$ be a map of systems inducing an isomorphism in $i n j-$ $H o\left(\mathcal{C}^{*}\right)$ and let $\underline{g}: \underline{B} \rightarrow \underline{A}$ be a map of systems inducing an homotopy inverse 
of $[\underline{f}]$. By reindexing (Lemma 1.11) we may assume that there is a homotopy commutative diagram

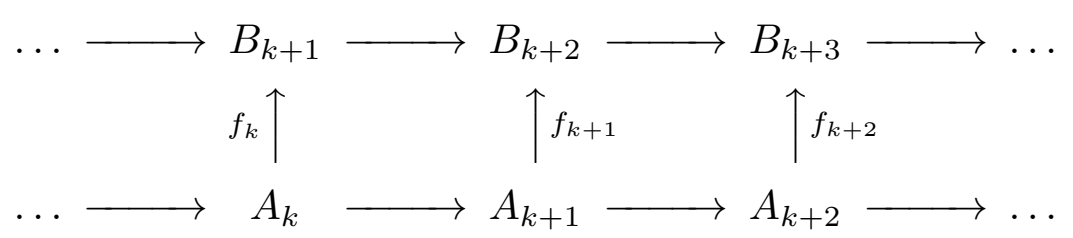

Take homotopies $h_{k}: A_{k} \rightarrow A_{k+1}[0,1]$ with $h_{k}^{0}=g_{k+1} f_{k}, h_{k}^{1}=p_{k+1 k}$ and $h_{k}^{\prime}: B_{k} \rightarrow B$ with $h_{k}^{\prime 0}=q_{k+1 k}, h_{k}^{\prime 1}=f_{k} g_{k}$. Let $\underline{C}$ be the inductive system

$$
\ldots \rightarrow A_{k} \stackrel{f_{k}}{\longrightarrow} B_{k+1} \stackrel{g_{k+1}}{\longrightarrow} A_{k+1} \stackrel{f_{k+1}}{\longrightarrow} B_{k+2} \stackrel{g_{k+2}}{\longrightarrow} \ldots
$$

We form the following commutative diagram

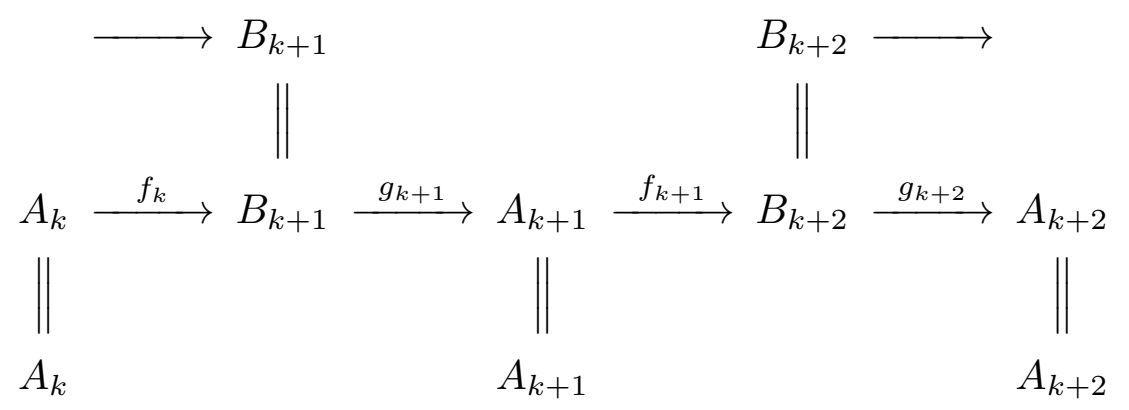

By Lemma $1.12 u=\left(i d_{A_{k}}, h_{k}\right): \underline{A} \rightarrow \underline{C}$ and $v=\left(i d_{B_{k}}, h_{k}^{\prime}\right): \underline{C} \rightarrow \underline{B}$ are isomorphisms in $\mathrm{Ho}\left(\operatorname{inj}_{-} \mathcal{C}^{*}\right)$. Hence $v u$ induces an isomorphism $\underline{A} \rightarrow \underline{B}$. Moreover it is clear from the above diagram that $[[v u]]$ is a lifting for $[f]$ "i.e." $F[[v u]]=[f]$.

\section{The homotopy inductive limit functor}

2.1 In this section we construct a functor $L: \operatorname{Ho}\left(i n j-\mathcal{C}^{*}\right) \rightarrow \mathcal{A}$ such that the following diagram is commutative:

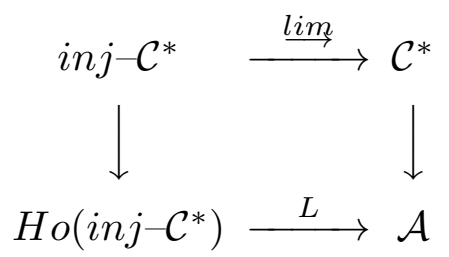

$L$ agrees with $\varliminf$ on objects that is $L(\underline{A})=\underline{\varliminf} \underline{A}=A$ for any inductive system of separable $\mathrm{C}^{*}$-algebras $\underline{A}=\left(A_{n}, p_{n+1 n}\right)$. Denote by $p_{n}$ the canonical mapping of $A_{n}$ into $A$. Next we define $\mathrm{L}$ on morphisms.

2.2 For $s \geq 1$ let $T_{s}=[s,+\infty)$ and let $C_{b}\left(T_{s}, B\right)$ denote the $\mathrm{C}^{*}$-algebra of continuous bounded functions from $T_{s}$ to $B$. Note that if $s(n)$ is an unbounded increasing sequence and $r_{n+1 n}: C_{b}\left(T_{s(n)}, B\right) \rightarrow C_{b}\left(T_{s(n+1)}, B\right)$ is the restriction map then $\lim _{(}\left(C_{b}\left(T_{s(n)}, B\right), r_{n+1 n}\right)=B_{\infty}$. The quotient map $C_{b}\left(T_{s}, B\right) \rightarrow B_{\infty}$ is denoted by $r_{s}$. For any continuous map $\eta: A \rightarrow C_{b}\left(T_{s}, B\right)$ we set $\hat{\eta}=r_{s} \circ \eta$. Recall that if $\eta$ is an asymptotic morphisms then sometimes we may use the notation $\eta_{t}$. This notation will be changed to $\eta^{t}$ whenever $\eta$ is known to be a $*$-homomorphism. 
Let $(\underline{f}, \underline{h}):\left(A_{n}, p_{n+1 n}\right) \rightarrow\left(B_{n}, q_{n+1 n}\right)$ be a strong map of inductive systems of $\mathrm{C}^{*}$-algebras.

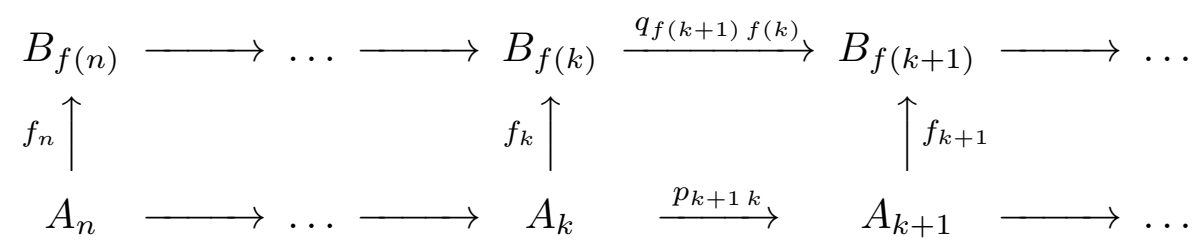

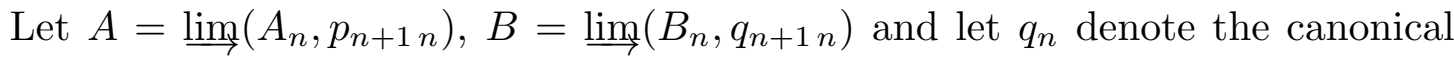
mapping of $B_{n}$ into $B$. We show now that there is an asymptotic morphism $\varphi_{t}$ : $A \rightarrow B$ naturally associated with $(\underline{f}, \underline{h})$. This will define a correspondence $(\underline{f}, \underline{h}) \rightarrow$ $\varphi_{t}=L_{0}((\underline{f}, \underline{h}))$. For any $n$ we define a $*$-homomorphism $\varphi_{n}: A_{n} \rightarrow C_{b}\left(\bar{T}_{n}, B\right)$, $\varphi_{n}=\left(\varphi_{n}^{t}\right)_{t \in T_{n}}$ by

$$
\varphi_{n}^{t}=q_{f(k+1)} h_{k}^{t-k} p_{k n}
$$

for $n \leq k \leq t \leq k+1$. Note that $\varphi_{n}$ is well defined since by the definition of strong maps of systems

$$
\begin{aligned}
& h_{k}^{0}=q_{f(k+1) f(k)} f_{k} \\
& h_{k}^{1}=f_{k+1} p_{k+1 k}
\end{aligned}
$$

Let $r_{n+1 n}: C_{b}\left(T_{n}, B\right) \rightarrow C_{b}\left(T_{n+1}, B\right)$ be the restriction map. We can assemble the *-homomorphisms $\varphi_{n}$ to get the following morphism of inductive systems

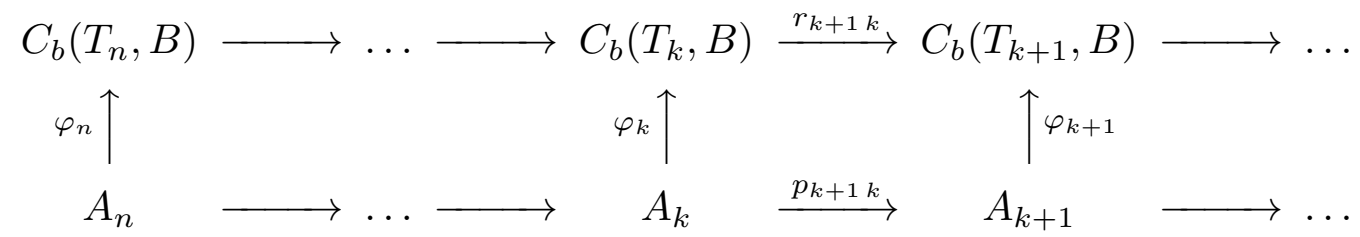

Via the inductive limit functor this induces a $*$-homomorphism

$$
\hat{\varphi}=\varliminf \varphi_{n}: \varliminf A_{n} \rightarrow \lim _{\longrightarrow} C_{b}\left(T_{n}, B\right)
$$

such that $\hat{\varphi} p_{n}=r_{n} \varphi_{n}$ where $r_{n}$ denotes the canonical mapping of $C_{b}\left(T_{k}, B\right)$ into $\varliminf_{(}\left(C_{b}\left(T_{n}, B\right), r_{n+1}\right) \cong B_{\infty} \cong C_{b}(T, B) / C_{0}(T, B)$. By definition we set

$$
L_{0}(\underline{f}, \underline{h})=\hat{\varphi} .
$$

Let $\varphi: A \rightarrow C_{b}(T, B)$ be a continuous lifting of $\hat{\varphi}$. By abuse of notation we will often write $L_{0}(f, \underline{h}) \cong \varphi_{t}$ to mean that $L_{0}(f, \underline{h})$ determines $\varphi_{t}$ up to an equivalence of asymptotic morphisms. We show now that $L_{0}$ passes to homotopy classes of strong maps of systems.

Lemma 2.3 If $(\underline{f}, \underline{h}),\left(\underline{f}^{\prime}, \underline{h}^{\prime}\right): \underline{A} \rightarrow \underline{B}$ are homotopic then $L_{0}(\underline{f}, \underline{h})$ is homotopic to $L_{0}\left(\underline{f}^{\prime}, \underline{h}^{\prime}\right)$.

Proof Assume first that $(\underline{f}, \underline{h})$ is is equivalent to $\left(\underline{f}^{\prime}, \underline{h}^{\prime}\right)$. This means that there is an increasing function $\nu: \mathbb{N} \rightarrow \mathbb{N}$ such that $q_{\nu(k) f(k+1)} h_{k}^{\tau}=q_{\nu(k) f^{\prime}(k+1)} h_{k}^{\prime \tau}$ (see 1.5). This implies $\varphi_{k}=\varphi_{k}^{\prime}$ for all $k$ and hence $\hat{\varphi}=\hat{\varphi}^{\prime}$.

Consider now the general case of two homotopic strong maps of systems. By Remark 1.8 and the previous discussion we may assume that the homotopy is 
implemented by a strong map $(\underline{\nu}, \underline{\mu}): \underline{A} \rightarrow \underline{B}[0,1]$ such that $\underline{e}^{0}(\underline{\nu}, \underline{\mu})$ is equal to $(\underline{f}, \underline{h})$ and $\underline{e}^{1}(\underline{\nu}, \underline{\mu})$ is equal to $\left(\underline{f}^{\prime}, \underline{h}^{\prime}\right)$. We claim that $\Phi \cong L_{0}(\underline{\nu}, \underline{\mu}): A \rightarrow$ $C_{b}(\bar{T}, B[0,1])$ is a homotopy from $\varphi_{t}$ to $\varphi_{t}^{\prime}$. Let $\phi_{n}: A_{n} \rightarrow C_{b}\left(T_{n}, \bar{B}[0,1]\right)$ be defined by

$$
\Phi_{n}^{t}=q_{f(k+1)} \otimes 1 \mu_{k}^{t-k} p_{k n} \quad \text { for } \quad n \leq k \leq t \leq k+1 .
$$

Since $\underline{e^{0}}(\underline{\nu}, \underline{\mu})$ is equal to $(\underline{f}, \underline{h})$ the diagram

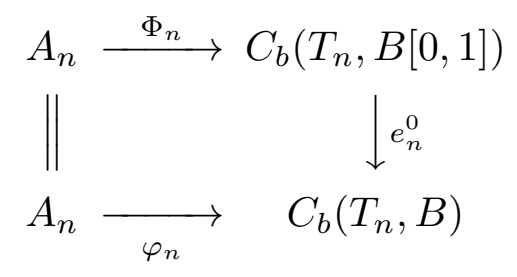

is commutative. This implies that $\hat{\Phi}^{0}=\hat{\varphi}$ and similarly $\hat{\Phi}^{1}=\hat{\varphi}^{\prime}$.

We define $L$ on morphisms $L:[[\underline{A}, \underline{B}]] \rightarrow[[A, B]]$ by $L[[(f, \underline{h})]]=\left[\left[L_{0}(\underline{f}, \underline{h})\right]\right]$. By Lemma 2.3 this is well defined. Next we show that $L$ preserves the composition of morphisms.

Theorem 2.4 $L: H o\left(i n j-\mathcal{C}^{*}\right) \rightarrow \mathcal{A}$ is a functor.

As a corollary of Theorems 1.14 and 2.4 we have the following

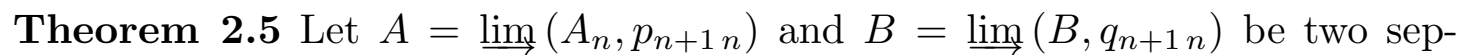
arable $C^{*}$-algebras. Suppose that the inductive systems $\left(A_{n}, p_{n+1 n}\right)$ and $B=$

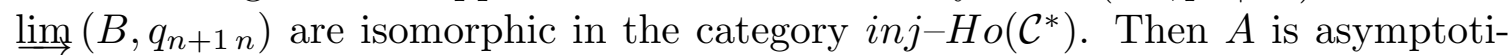
cally homotopy-equivalent to $B$, i.e. $A$ is isomorphic to $B$ in the category $\mathcal{A}$.

Theorem 2.5 shows that the homotopy classes of the connecting maps of an inductive system of $\mathrm{C}^{*}$-algebras determine the inductive limit up to an asymptotic homotopy equivalence.

For the proof of Theorem 2.4 we need the following construction.

2.6 Given an increasing map $\alpha: \mathbb{N} \rightarrow \mathbb{N}$ and a strong map of systems $(\underline{f}, \underline{h})$ we define the strong map of systems $\left(f^{\alpha}, \underline{h}^{\alpha}\right)$ by:

The index map $f^{\alpha}: \mathbb{N} \rightarrow \mathbb{N}, f^{\alpha}=\bar{f} \circ \alpha$.

The $*$-homomorphisms $f_{n}^{\alpha}=f_{\alpha(n)} p_{\alpha(n) n}$.

The homotopies $h_{n}^{\alpha}=h_{\alpha, n}$ (see 1.6 ).

It is easy to see that $\left(\underline{f}^{\alpha}, \underline{h}^{\alpha}\right)$ is homotopic to $(\underline{f}, \underline{h}) \circ i_{\alpha}$ hence homotopic to $(\underline{f}, \underline{h})$ (see 1.10-1.11). Let $\tilde{\alpha}: T \rightarrow T$ be an extension of $\alpha$ given by $\tilde{\alpha}(t)=(1-t+k) \alpha(k)+(t-k) \alpha(k+1)$ for $k \leq t \leq k+1$.

Lemma If $L_{0}(\underline{f}, \underline{h}) \cong \varphi_{t}$ then $L_{0}\left(\underline{f}_{\alpha}, \underline{h}_{\alpha}\right) \cong L_{0}\left(\underline{f}^{\alpha}, \underline{h}^{\alpha}\right) \cong \varphi_{\tilde{\alpha}(t)}$.

2.7 (Proof of Theorem 2.4) The proof has four parts (a)-(d). (a) Let $(\underline{f}, \underline{h}): \underline{A} \rightarrow \underline{B}$ and $\left(\underline{f}^{\prime}, \underline{h}^{\prime}\right): \underline{B} \rightarrow \underline{C}$. Consider the corresponding asymptotic morphisms $L_{0}(\underline{f}, \underline{h}) \cong \varphi: A \rightarrow C_{b}(T, B)$

and $L_{0}\left(f^{\prime}, \underline{h}^{\prime}\right) \cong \varphi^{\prime}: B \rightarrow C_{b}(T, C)$. These are (continuous) liftings of $\hat{\varphi}: A \rightarrow B_{\infty}$ and $\hat{\varphi}^{\prime}: B \rightarrow C_{\infty}$ hence are unique up to an equivalence. Recall that by definition

$$
\hat{\varphi} p_{n}=r_{n} \varphi_{n}, \quad \hat{\varphi}^{\prime} p_{n}=r_{n}^{\prime} \varphi_{n}^{\prime}
$$


where $r_{n}$ is the canonical map from $C_{b}\left(T_{n}, B\right)$ to $B_{\infty}$ and $r_{n}^{\prime}$ is the canonical map from $C_{b}\left(T_{n}, C\right)$ to $C_{\infty}$. This implies

$$
\lim _{t \rightarrow \infty}\left\|\varphi_{t}\left(p_{n}(a)\right)-\varphi_{n}^{t}(a)\right\|=0 \quad \lim _{t \rightarrow \infty}\left\|\varphi_{t}^{\prime}\left(q_{n}(b)\right)-\varphi_{n}^{\prime t}(b)\right\|=0
$$

for all $a \in A_{n}$ and $b \in B_{n}$.

We construct inductively two sequences of compacts $K_{n} \subset A_{n}$ and $\hat{K}_{n} \subset A$ such that $p_{n+1 n}\left(K_{n}\right) \subset K_{n+1}, p_{n}\left(K_{n}\right) \subset \hat{K}_{n}, \hat{K}_{n} \subset \hat{K}_{n+1}$ and the union of $p_{n}\left(K_{n}\right)$ is a dense selfadjoint subalgebra of $A$. Moreover we can take the compacts $\hat{K}_{n}$ such that $\hat{K}_{n}+\hat{K}_{n} \subset \hat{K}_{n+1}, \hat{K}_{n} \hat{K}_{n} \subset \hat{K}_{n+1}$, and $\lambda \hat{K}_{n} \subset \hat{K}_{n}$ for all $\lambda \in \mathbb{C}$ with $|\lambda| \leq 1$ $[\mathrm{CH}]$. By (5) one can find an increasing map $\alpha: \mathbb{N} \rightarrow \mathbb{N}$ such that

$$
\left\|\varphi_{t}\left(p_{n}(x)\right)-\varphi_{n}^{t}(x)\right\| \leq 1 / n, \quad \text { for all } x \in K_{n}, t \geq \alpha(n)
$$

Since $\varphi_{t}$ is uniformly continuous on compacts for a suitable fast increasing map $\alpha$ we can arrange that

$$
\begin{aligned}
\left\|\varphi_{t}(a+\lambda b)-\varphi_{t}(a)-\lambda \varphi_{t}(b)\right\| & \leq 1 / n \\
\left\|\varphi_{t}(a b)-\varphi_{t}(a) \varphi_{t}(b)\right\| & \leq 1 / n \\
\left\|\varphi_{t}\left(a^{*}\right)-\varphi_{t}(a)^{*}\right\| & \leq 1 / n \\
\left\|\varphi_{t}(a)\right\| & \leq\|a\|+1 / n
\end{aligned}
$$

for all $t \geq \alpha(n), a, b \in \hat{K}_{n}$ and $|\lambda| \leq 1$ (cf. $\left.[\mathrm{CH}]\right)$.

By replacing $(\underline{f}, \underline{h})$ by $\left(\underline{f}^{\alpha}, \underline{h}^{\alpha}\right)$ and $\varphi_{t}$ by $\varphi_{\tilde{\alpha}(t)}$ we may assume that (6)-(10) hold true with $\alpha(n)=n$ (see Lemma 2.6). It is convenient to reindex the inductive system $\underline{B}$ such that the indexing map of $(\underline{f}, \underline{h})$ becomes the identity map of $\mathbb{N}$. This is achieved by replacing $\left(\underline{f}^{\prime}, \underline{h}^{\prime}\right)$ by $\left(\underline{f}_{f}^{\prime}, \underline{h}_{f}^{\prime}\right)$ (see 1.6) and $\varphi_{t}^{\prime}$ by $\varphi_{\tilde{f}(t)}$.

Next we construct inductively two more sequences of compacts $K_{n}^{\prime} \subset B_{n}$ and $\hat{K}_{n}^{\prime} \subset B$ such that $q_{n+1 n}\left(K_{n}^{\prime}\right) \subset K_{n+1}^{\prime}, q_{n+1}\left(K_{n+1}^{\prime}\right) \subset \hat{K}_{n}^{\prime}$ and $\hat{K}_{n}^{\prime} \subset \hat{K}_{n+1}^{\prime}$. Moreover we can take the compacts $K_{n}^{\prime}, \hat{K}_{n}^{\prime}$ such that

$$
\begin{aligned}
& \left\{h_{n}^{\tau}(x): x \in K_{n}, \tau \in[0,1]\right\} \subset K_{n+1}^{\prime} \\
& \left\{\varphi_{t}(x): x \in \hat{K}_{n+3}, 1 \leq t \leq n+1\right\} \subset \hat{K}_{n}^{\prime}
\end{aligned}
$$

By replacing $\left(\underline{f}^{\prime}, \underline{h}^{\prime}\right)$ by $\left(f^{\prime \beta}, h^{\prime \beta}\right)$ and $\varphi_{t}^{\prime}$ by $\varphi_{\tilde{\beta}(t)}^{\prime}$ for a fast increasing function $\beta: \mathbb{N} \rightarrow \mathbb{N}$, we may assume that

$$
\left\|\varphi_{t}^{\prime}\left(q_{n}(y)\right)-\varphi_{n}^{\prime t}(y)\right\| \leq 1 / n, \quad \text { for all } y \in K_{n}^{\prime}, t \geq n
$$

$$
\begin{aligned}
\left\|\varphi_{t}^{\prime}(a+\lambda b)-\varphi_{t}^{\prime}(a)-\lambda \varphi_{t}^{\prime}(b)\right\| & \leq 1 / n \\
\left\|\varphi_{t}^{\prime}(a b)-\varphi_{t}^{\prime}(a) \varphi_{t}^{\prime}(b)\right\| & \leq 1 / n \\
\left\|\varphi_{t}^{\prime}\left(a^{*}\right)-\varphi_{t}^{\prime}(a)^{*}\right\| & \leq 1 / n \\
\left\|\varphi_{t}^{\prime}(a)\right\| & \leq\|a\|+1 / n .
\end{aligned}
$$


for all $t \geq n, a, b \in \hat{K}_{n}^{\prime}$ and $|\lambda| \leq 1$.

(b) Having (7)-(10) and (12)-(16) it follows as in Lemma 3 in $[\mathrm{CH}]$ that $\varphi_{t+1}^{\prime} \circ \varphi_{t}$ is an asymptotic morphism on the union of $\hat{K}_{n}$. Its extension to $A$ is an asymptotic morphism (well defined up to an equivalence) whose homotopy class is equal to the composition $\left[\left[\varphi_{t}^{\prime}\right]\right] \circ\left[\left[\varphi_{t}\right]\right]$.

On the other hand we claim that the product $\left[\left[f^{\prime}, \underline{h}^{\prime}\right]\right] \circ[[f, \underline{h}]]$ is given by the homotopy class of $\left(f^{\prime \prime}, \underline{h}^{\prime \prime}\right)$ where

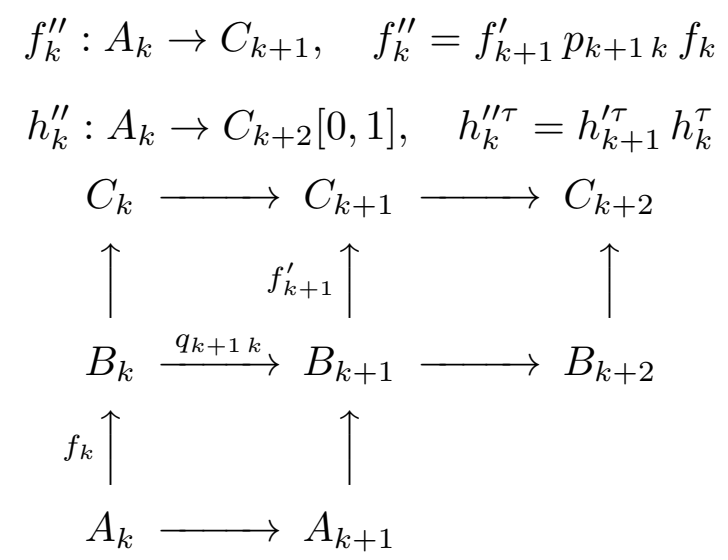

Indeed for $\alpha(k)=k+1$ we have

$$
\left[\left[\underline{f}^{\prime}, \underline{h}^{\prime}\right]\right] \circ[[\underline{f}, \underline{h}]]=\left[\left[\underline{f}^{\prime \alpha}, \underline{h}^{\prime \alpha}\right]\right] \circ[[\underline{f}, \underline{h}]]=\left[\left[\underline{f}^{\prime \prime}, \underline{g}^{\prime \prime}\right]\right]
$$

where according to $1.7 \underline{g}^{\prime \prime}$ is given by the sequence of homotopies

$$
g_{k}^{\prime \prime \tau}=\left\{\begin{array}{lll}
h_{k+1}^{2 \tau} q_{k+1 k} f_{k}, & \text { if } \quad 0 \leq \tau \leq 1 / 2 \\
f_{k+1} q_{k+2 k+1} h_{k}^{2 \tau-1}, & \text { if } \quad 1 / 2 \leq \tau \leq 1
\end{array}\right.
$$

Finally it is easily seen that $\left(\underline{f}^{\prime \prime}, \underline{g}^{\prime \prime}\right)$ is homotopic to $\left(\underline{f}^{\prime \prime}, \underline{h}^{\prime \prime}\right)$ since each $g_{k}^{\prime \prime}$ is homotopic to $h_{k}^{\prime \prime}$ canonically.

According to the definition of $L_{0}$ the $*$-homomorphism $\hat{\varphi}^{\prime \prime}=L_{0}\left(f^{\prime \prime}, \underline{h}^{\prime \prime}\right)$ is the inductive limit of the sequence of $*$-homomorphisms $\varphi_{n}^{\prime \prime}: A_{n} \rightarrow C_{b}\left(T_{n}, C\right)$ given by

$$
\varphi_{n}^{\prime \prime t}=r_{k+2} h_{k+1}^{t-k} h_{k}^{t-k} p_{k+1 k}
$$

for all $t$ with $n \leq k \leq t \leq k+1$.

(c) We claim that the proof of Theorem 2.4 is finished once we show that

$$
\left\|\varphi_{n}^{\prime \prime}(x)-\varphi_{t+1}^{\prime} \varphi_{t}\left(p_{n}(x)\right)\right\| \leq 4 / n .
$$

for all $x \in K_{n}$ and $n \leq t \leq n+1$.

Indeed we show below that (18) implies

$$
\lim _{t \rightarrow \infty}\left\|\varphi_{t}^{\prime \prime}(a)-\varphi_{t+1}^{\prime} \varphi_{t}(a)\right\|=0
$$

for all $a \in A$. Since the union of $p_{n}\left(K_{n}\right)$ is dense in $A$ it is enough to prove (19) only for elements $a=p_{n}(x)$ with $x \in K_{n}$. Moreover since $\lim _{t \rightarrow \infty}\left\|\varphi_{t}^{\prime \prime}\left(p_{n}(x)\right)-\varphi_{n}^{\prime \prime t}(x)\right\|=0$ it is sufficient to show that

$$
\lim _{t \rightarrow \infty}\left\|\varphi_{n}^{\prime \prime t}(x)-\varphi_{t+1}^{\prime} \varphi_{t}\left(p_{n}(x)\right)\right\|=0 .
$$


Let $n \leq k \leq t \leq k+1$ and set $z=p_{k n}(x) \in K_{k}$. According to (18)

$$
\left\|\varphi_{k}^{\prime \prime t}(z)-\varphi_{t+1}^{\prime} \varphi_{t}\left(p_{k}(z)\right)\right\| \leq 4 / k
$$

But $\varphi_{k}^{\prime \prime t}(z)=\varphi_{k}^{\prime \prime t}\left(p_{k n}(x)\right)=\varphi_{n}^{\prime \prime t}(x)$ for all $t \geq k$.

Thus $\left\|\varphi_{n}^{\prime \prime t}(x)-\varphi_{t+1}^{\prime} \varphi_{t}\left(p_{n}(x)\right)\right\| \leq 4 / k$.

This implies (20) and therefore (19).

(d) In the last part of the proof we show that (18) holds true. As a first step we show that

$$
\left\|\varphi_{t+1}^{\prime}\left(\varphi_{n}^{t}(x)\right)-\varphi_{t+1}^{\prime}\left(\varphi_{t}\left(p_{n}(x)\right)\right)\right\| \leq 3 / n
$$

for all $x \in K_{n}$ and $n \leq t \leq n+1$.

If $x \in K_{n}$ then $h_{n}^{\tau}(x) \in K_{n+1}^{\prime}$ for all $0 \leq \tau \leq 1$ by (11). Thus $\varphi_{n}^{t}(x)=$ $q_{n+1} h_{n}^{t-n}(x) \in \hat{K}_{n}^{\prime}$ since $q_{n+1}\left(K_{n+1}^{\prime}\right) \subset \hat{K}_{n}^{\prime}$. By $(12) \varphi_{t}\left(p_{n}(x)\right) \in \hat{K}_{n}^{\prime}$ since $p_{n}(x) \in \hat{K}_{n} \subset \hat{K}_{n+3}$. Since both $u=\varphi_{n}^{t}(x)$ and $v=\varphi_{t}\left(p_{n}(x)\right)$ belong to $\hat{K}_{n}^{\prime}$ by using (14), (17) and (6)

$$
\begin{gathered}
\left\|\varphi_{t+1}^{\prime}(u)-\varphi_{t+1}^{\prime}(v)\right\| \leq\left\|\varphi_{t+1}^{\prime}(u-v)\right\|+1 / n \leq \\
\|u-v\|+2 / n \leq 3 / n
\end{gathered}
$$

which proves (21).

Next we want to show that

$$
\left\|\varphi_{n}^{\prime \prime t}(x)-\varphi_{t+1}^{\prime}\left(\varphi_{n}^{t}(x)\right)\right\| \leq 1 / n
$$

for all $x \in K_{n}$ and $n \leq t \leq n+1$.

Let $y=h_{n}^{t-n}(x)$ and note that $y \in K_{n+1}^{\prime} \subset B_{n+1}$ by (11). It follows from (13) that

$$
\left\|\varphi_{t+1}^{\prime}\left(q_{n+1}(y)\right)-\varphi_{n+1}^{\prime t+1}(y)\right\| \leq 1 /(n+1)
$$

Recall that $\varphi_{n}^{t}(x)=q_{n+1} h_{n}^{t-n}(x)=q_{n+1}(y)$. Recall that $\varphi_{n}^{\prime \prime t}(x)=r_{n+2}^{\prime} h_{n+1}^{\prime t-n} h_{n}^{t-n}(x)$. This is equal to $\varphi_{n+1}^{t+1}(y)$ by the definition of $\varphi_{n+1}^{\prime}$. Thus (22) follows from (23).

Finally we obtain (18) as a consequence of (21) and (22).

\section{The strong shape category}

Definition $3.1([\mathrm{~B}])$ Let $A, B$ be separable $\mathrm{C}^{*}$-algebras. A *-homomorphism $\varphi: A \rightarrow B$ is called semiprojective if for any $\mathrm{C}^{*}$-algebra $E$ and increasing sequence $J_{n}$ of ideals of $E$ with $J=\overline{\cup J_{n}}$ and for any $*$-homomorphism $\sigma: B \rightarrow E / J$ there is an $n$ and a $*$-homomorphism $\psi: A \rightarrow E / J_{n}$ with $\pi_{n} \psi=\sigma \varphi$ where $\pi_{n}: E / J_{n} \rightarrow E / J$ is the quotient map.

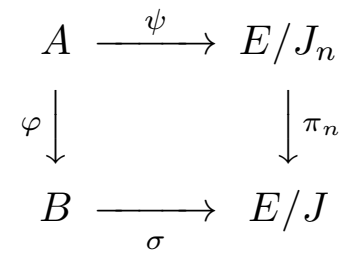


$A$ is semiprojective if the identity map of $A$ is semiprojective. A composition of a semiprojective $*$-homomorphism with any other $*$-homomorphism is semiprojective. The reader is refered to [EK1], [B] and [Lo] for more discussion on semiprojectivity.

Definition 3.2 An inductive system $\left(A_{n}, p_{n+1 n}\right)$ of separable $\mathrm{C}^{*}$-algebras is called semiprojective if all the $*$-homomorphisms $p_{n+1} n$ are semiprojective.

3.3 It is proven in $[\mathrm{B}]$ that any $\mathrm{C}^{*}$-algebra can be written as the inductive limit of some semiprojective inductive system. The shape category $\mathcal{S} h$ is the full subcategory of $i n j-H o\left(\mathcal{C}^{*}\right)$ whose objects are all the semiprojective inductive systems. The shape invariant of a separable $\mathrm{C}^{*}$-algebra $A$ is the isomorphism class in $\mathcal{S} h$ of some projective system $\underline{A}$ with $\underline{\underline{\lim }} \underline{A}=A$. This is well defined for if $\underline{A}^{\prime}$ is another semiprojective system with $\underline{\underline{A^{\prime}}} \underline{A}^{\prime}=A$, then $\underline{A}^{\prime}$ is isomorphic to $\underline{A}$ in $\mathcal{S} h$ (see Theorem 4.8 in $[\mathrm{B}]$ ). Two separable $\mathrm{C}^{*}$-algebras are said to be shape equivalent if and only if they have the same shape invariant. The shape invariant of a $\mathrm{C}^{*}$-algebra is a homotopy invariant. In general the shape invariant of a $\mathrm{C}^{*}$-algebra $A$ does not determine the homotopy type of $A$. However, according to Theorem 2.5, it determines the "asymptotic homotopy type" of $A$. We shall prove that the converse is also true (see Theorem 3.9 below).

3.4 It is convenient to extend the shape invariant of [B] to a shape functor $S h: \mathcal{C}^{*} \rightarrow \mathcal{S} h$. To this purpose for any separable $\mathrm{C}^{*}$-algebra $A$ we choose a semiprojective system $\underline{A}$ such that $\underline{\varliminf} \underline{A}=A$ (The Choice Axiom is invoked). By using arguments similar to those in Section 5 of [EK1] and Theorem 3.3 of [B] for each $*$-homomorphism $\varphi: A \rightarrow B$ one finds a morphism $\underline{f}: \underline{A} \rightarrow \underline{B}$ such that the diagram

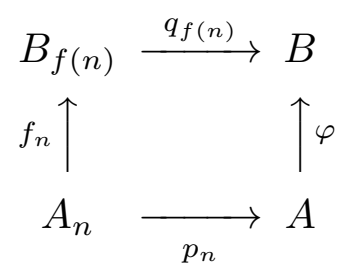

commutes up to a homotopy, for all $n$. The morphism $f$ is unique up to homotopy. The shape functor associated with the selection $A \rightsquigarrow \underline{A}$ is defined on objects by $S h(A)=\underline{A}$ and on $*$-homomorphisms by $S h(\varphi)=[f]$. If two $*$-homomorphisms $\varphi, \psi: A \rightarrow B$ are homotopic then $\operatorname{Sh}(\varphi)=S h(\psi)$ and hence the shape functor descends to a functor

$$
S h: H o\left(\mathcal{C}^{*}\right) \rightarrow \mathcal{S} h \subset i n j-H o\left(\mathcal{C}^{*}\right) .
$$

It is easily seen that different selections $A \rightsquigarrow \underline{A}$ give rise to naturally isomorphic shape functors.

Theorem 3.5 Let $\underline{A}=\left(A_{n}, p_{n+1 n}\right)$ and $\underline{B}=\left(B_{n}, q_{n+1 n}\right)$ be inductive systems of separable $C^{*}$-algebras and let $A=\underline{\varliminf} \underline{A}, B=\underline{\varliminf} \underline{B}$. Suppose that $\underline{A}$ is semiprojective. Then the homotopy inductive limit functor induces a bijection

$$
L:[[\underline{A}, \underline{B}]] \rightarrow[[A, B]] .
$$

Proof This follows from Corollary 3.15 and Proposition 3.18 given below. 
Motivated by Theorem 3.5 we introduce the strong shape category of separable $\mathrm{C}^{*}$-algebras. This is a generalization of topological strong shape category ( see $\mathrm{CH}$. III 9 in [MS] for a survey of the commutative theory and additional references). A good reference for the strong shape theory of compact metrisable spaces is [Ca].

Definition 3.6 The strong shape category of separable $\mathrm{C}^{*}$-algebras, denoted by $\mathcal{S}$, is the full subcategory of $H o\left(i n j-\mathcal{C}^{*}\right)$ whose objects are all the semiprojective inductive systems. Two separable $\mathrm{C}^{*}$-algebras $A$ and $B$ are said to be strong shape equivalent if there are semiprojective inductive systems $\underline{A}$ and $\underline{B}$ such that $\underline{\varliminf} \underline{A}=A, \underline{\varliminf} \underline{B}=B$ and $\underline{A}$ is isomorphic to $\underline{B}$ in $\mathcal{S}$. Using the Choice Axiom we select for each separable $\mathrm{C}^{*}$-algebra $A$ a semiprojective system $\underline{A}$ with $\underline{\lim } \underline{A}=A$. By Theorem 3.5 for each $w \in[[A, B]]$ there is a unique $u \in[[\underline{A}, \underline{B}]]$ such that $L u=w$. A strong shape functor $S: \mathcal{A} \rightarrow \mathcal{S}$ is defined by setting $S(A)=\underline{A}$ and $S(w)=u$. Different selections $A \rightsquigarrow \underline{A}$ give rise to naturally isomorphic strong shape functors.

The restriction of the homotopy inductive limit functor $L$ to the strong shape category $\mathcal{S}$ is denoted by $L$ too.

Theorem 3.7 The functor $L: \mathcal{S} \rightarrow \mathcal{A}$ is an equivalence of categories. The strong shape functor $S$ is a right inverse for $L$.

Proof The Theorem follows from Theorem 3.5 and Definition 3.6

Let $\mathcal{K}$ denote the $\mathrm{C}^{*}$-algebra of compact operators on a separable infinite dimensional Hilbert space. The suspension of a $\mathrm{C}^{*}$-algebra $A$ is $S A=C_{0}(\mathbb{R}) \otimes A$. The E-theory group was defined in $[\mathrm{CH}]$ by $E(A, B)=[[S A \otimes \mathcal{K}, S B \otimes \mathcal{K}]]$.

Corollary 3.8 E-theory is equivalent to the strong shape theory of suspensions of stable separable $\mathrm{C}^{*}$-algebras.

Theorem 3.9 Let $A, B$ be separable $\mathrm{C}^{*}$-algebras. The following are equivalent:

(a) $A$ is shape equivalent to $B$.

(b) $A$ is strong shape equivalent to $B$.

(c) $A$ is isomorphic to $B$ in $\mathcal{A}$ i.e. there are asymptotic morphisms

$\varphi_{t}: A \rightarrow B$ and $\psi_{t}: B \rightarrow A$ such that $\left[\left[\psi_{t}\right]\right] \circ\left[\left[\varphi_{t}\right]\right]=\left[\left[i d_{A}\right]\right]$ and $\left[\left[\varphi_{t}\right]\right] \circ\left[\left[\psi_{t}\right]\right]=\left[\left[i d_{B}\right]\right]$.

Proof The Theorem is a straightforward consequence of Theorems 1.14 and 3.7

Remark 3.10 Let $F: \mathcal{S} \rightarrow \mathcal{S} h$ be the restriction of the canonical functor $F: H o\left(i n j-\mathcal{C}^{*}\right) \rightarrow i n j-H o\left(\mathcal{C}^{*}\right)($ see 1.13$)$. The diagram

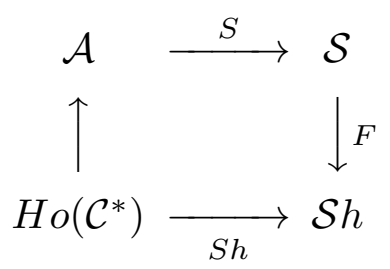

is commutative up to a natural isomorphism of functors. Thus the shape functor factors through $\mathcal{A}$. 
Let $\mathcal{C}$ be a category. An inductive system $\left(A_{n}, p_{n+1 n}\right)$ in $\mathcal{C}$ consists of a infinite diagram

$$
\ldots \rightarrow A_{n} \stackrel{p_{n+1 n}}{\longrightarrow} A_{n+1} \rightarrow \ldots
$$

of objects and morphisms in $\mathcal{C}$. Recall that a couple $\left(A, p_{n}\right)$ consisting of an object $A$ of $\mathcal{C}$ and a sequence of morphisms $p_{n} \in \operatorname{Hom}_{\mathcal{C}}\left(A_{n}, A\right)$ is an inductive limit of the system $\left(A_{n}, p_{n+1 n}\right)$ if the following two conditions are fullfilled.

1) $p_{n+1} p_{n+1 n}=p_{n}$

2) For any couple $\left(B, q_{n}\right)$ consisting of an object $B$ of $\mathcal{C}$ and a sequence of morphisms $q_{n} \in \operatorname{Hom}_{\mathcal{C}}\left(A_{n}, B\right)$ such that $q_{n+1} p_{n+1 n}=q_{n}$, there is a unique $\varphi \in$ $H \operatorname{Hom}_{\mathcal{C}}(A, B)$ such that $\varphi p_{n}=q_{n}$ for all $n$.

Let $\mathcal{B}$ be a category. We say that a covariant functor $T: \mathcal{C} \rightarrow \mathcal{B}$ preserves inductive limits if whenever $\left(A, p_{n}\right)$ is an inductive limit of a system $\left(A_{n}, p_{n+1 n}\right)$, $\left(T(A), T\left(p_{n}\right)\right)$ is an inductive limit of the system $\left(T\left(A_{n}\right), T\left(p_{n+1 n}\right)\right)$.

Theorem 3.11 Let $\mathcal{B}$ be a category and let $T: \mathcal{C}^{*} \rightarrow \mathcal{B}$ be a homotopy invariant functor. If $T$ preserves inductive limits then $T$ factors through the category $\mathcal{A}$. In particular $T(S A \otimes \mathcal{K})$ is naturally isomorphic to $T\left(S^{3} A \otimes \mathcal{K}\right)$ for any separable $\mathrm{C}^{*}$-algebra $A$ (cf. $[\mathrm{Cu}]$ ).

Proof Since $T$ is homotopy invariant and preserves inductive limits it is not hard to see that $T$ extends canonically to a functor $T^{\prime}: i n j-H o(\mathcal{C}) \rightarrow \mathcal{B}$ such that $T=T^{\prime} \circ S h$. Thus the first part of the statement follows from Remark 3.10. The second part of the statement becomes a consequence of the Bott periodicity in E-theory: $S \mathcal{K}$ is asymptotically homotopy-equivalent to $S^{3} \mathcal{K}[\mathrm{CH}]$. [RS].

Let $\mathcal{N}$ be the category of "nice" separable nuclear $\mathrm{C}^{*}$-algebras introduced in

Proposition 3.12 Let $A, B$ be $\mathrm{C}^{*}$-algebras in $\mathcal{N}$. Then $S A \otimes \mathcal{K}$ is shape equivalent to $S B \otimes \mathcal{K}$ if and only if $K_{*}(A)$ is isomorphic to $K_{*}(B)$ as $\mathbb{Z} / 2$-graded groups.

Proof By the universal coefficient theorem of $[\mathrm{RS}], K_{*}(A) \cong K_{*}(B)$ if and only if $A$ is $K K$-equivalent to $B$. For nuclear $\mathrm{C}^{*}$-algebras $K K(A, B)$ is isomorphic to $E(A, B)=[[S A \otimes \mathcal{K}, S B \otimes \mathcal{K}]]$ by an isomorphism that preserves the intersection product $[\mathrm{CH}]$. The result follows from Theorem 3.9.

Many other shape classification results can be obtained based on results in [D1],[D2], [DL]. For instance we prove in [D3] that if $X$ and $Y$ are compact connected metrisable spaces then $C_{0}(X \backslash p t) \otimes \mathcal{K}$ is shape equivalent to $C_{0}(Y \backslash p t) \otimes \mathcal{K}$ if and only if $K^{*}(X)$ is isomorphic to $K^{*}(Y)$ as $\mathbb{Z} / 2$-graded groups.

The rest of the paper is devoted to a proof of Theorem 3.5.

Proposition 3.13 Let $\gamma: A \rightarrow B$ be a semiprojective $*$-homomorphism and let $E, J_{n}, J, \pi_{n}$ be as in Definition 3.1. Suppose that there are given two *homomorphisms $\alpha, \beta: B \rightarrow E / J_{n}$ and a homotopy $h: B \rightarrow E / J[0,1]$ from $\pi_{n} \alpha$ to $\pi_{n} \beta$. Then there are $m \geq n$ and a homotopy $h_{m}: A \rightarrow E / J_{m}[0,1]$ lifting the homotopy $h \circ \gamma\left(\right.$ i.e. $\left.\pi_{m} h_{m}^{t}=h^{t} \gamma\right)$ such that

$$
h_{m}^{0}=\pi_{m n} \alpha \gamma, \quad h_{m}^{1}=\pi_{m n} \beta \gamma .
$$

Here $\pi_{m n}: E / J_{n} \rightarrow E / J_{m}$ denotes the quotient map. 
Proof This is a version of Theorem 3.3 in [B]; (see also Proposition 3.2 in [EK1].)

Proposition 3.14 Let $\left(A_{n}, p_{n+1 n}\right)$ be a semiprojective inductive system, let

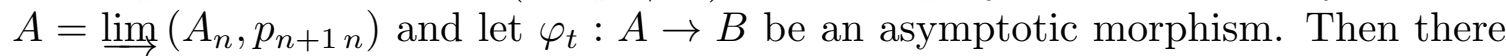
are an increasing map $\alpha: \mathbb{N} \rightarrow \mathbb{N}$, a sequence of $*$-homomorphisms $\varphi_{n}: A_{n} \rightarrow$ $C_{b}\left(T_{\alpha(n)}, B\right)$ such that $\varphi_{n}^{t}=\varphi_{n+1}^{t} p_{n+1 n}$ for all $t \geq \alpha(n)$ and $\hat{\varphi}_{n}=\hat{\varphi} \circ p_{n}$ for all $n$.

That means that there is a commutative diagram of $\mathrm{C}^{*}$-algebras and $*$-homomorphisms

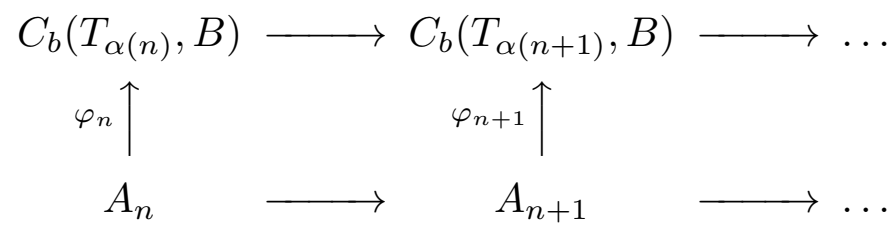

such that $\hat{\varphi}=\underline{\lim _{n}} \varphi_{n}$.

Proof Let $K_{n} \subset A_{n}$ be a sequence of compacts with $p_{n+1}{ }_{n}\left(K_{n}\right) \subset K_{n+1}$ and such that the union of $p_{n}\left(K_{n}\right)$ is a dense subset of $A$. We shall construct inductively

a sequence $\alpha(1)<\alpha(2)<\ldots<\alpha(n)$,

*-homomorphisms $\psi_{j}: A_{j+1} \rightarrow C_{b}\left(T_{\alpha(j)}, B\right)$ and $\eta_{j}: A_{j} \rightarrow C_{b}\left(T_{\alpha(j)}, B\right)$,

$\eta_{j}=\psi_{j} \circ p_{j+1 j}, 1 \leq j \leq n$

*-homomorphisms (homotopies) $H_{j}: A_{j} \rightarrow C_{b}\left(T_{\alpha(j+1)}, B[0,1]\right), 1 \leq j \leq n-1$

such that for all $j \leq n$ we have

$$
\begin{gathered}
\hat{\psi}_{j}=\hat{\varphi} p_{j+1} \\
\left\|\varphi_{t}\left(p_{j}(x)\right)-\eta_{j}^{t}(x)\right\| \leq 1 / j, \quad \text { for all } \quad x \in K_{j}, \quad t \geq \alpha(j) \\
\sup _{r, s \in[0,1]}\left\|H_{j-1}^{r, t}(x)-H_{j-1}^{s, t}(x)\right\| \leq 1 / j \quad \text { for all } \quad x \in K_{j}, \quad t \geq \alpha(j) . \\
H_{j-1}^{0, t}=\eta_{j-1}^{t}, \quad H_{j-1}^{1, t}=\eta_{j}^{t} p_{j-1} \quad \text { for all } \quad t \geq \alpha(j) .
\end{gathered}
$$

Suppose that $\alpha(j), \psi_{j}, \eta_{j}, H_{j-1}$ have been constructed for all $j \leq n$. We proceed now to producing $\alpha(n+1), \psi_{n+1}, \eta_{n+1}, H_{n}$.

Consider the $*$-homomorphism $\hat{\varphi} \circ p_{n+3}: A_{n+3} \rightarrow B_{\infty}$. Since $p_{n+3 n+2}$ is semiprojective there is a $*$-homomorphism $\psi_{n+1}: A_{n+2} \rightarrow C_{b}\left(T_{m}, B\right)$ for some big enough $m$ such that $\hat{\psi}_{n+1}=\hat{\varphi} \circ p_{n+2}$. Define $\eta_{n+1}=\psi_{n+1} \circ p_{n+2 n+1}$. Since $\hat{\psi}_{n}=\hat{\psi}_{n+1} p_{n+2 n+1}$ and $p_{n+1 n}$ is semiprojective we can use Proposition 3.13 to find a $*$-homomorphism $H_{n}: A_{n} \rightarrow C_{b}\left(T_{m}, B[0,1]\right)$ such that

$$
H_{n}^{0, t}=\psi_{n}^{t} \circ p_{n+1 n}=\eta_{n}^{t}, \quad H_{n}^{1, t}=\psi_{n+1}^{t} p_{n+2 n}=\eta_{n+1}^{t} p_{n+1 n} \quad \text { for all } t \geq m .
$$

Since $\hat{H}_{n}=\left(H_{n}^{s}\right)_{s \in[0,1]}$ lifts the constant homotopy $\hat{\psi}_{n} p_{n+1 n}$, it follows that (26) holds for $j=n+1$ and big enough $\alpha(n+1) \geq m$. It is clear that $\hat{\eta}_{n}=\hat{\varphi} p_{n}$ and hence by increasing $\alpha(n+1)$ we can arrange to have (2.5) fulfilled for $j=n+1$.

In the last part of the proof we produce the $*$-homomorphisms $\varphi_{n}$ inductively. For any positive integer $k \geq n$ we can find $\beta(k) \in[\alpha(k), \alpha(k+1)]$ and an increasing 
homeomorphism $u:[\alpha(k), \alpha(k+1)] \rightarrow[\alpha(k), \beta(k)]$ such that $\| \eta_{n}^{u(t)}\left(p_{k n}\right)(x)-$ $\eta_{n}^{t}\left(p_{k n}(x) \| \leq 1 / k\right.$ for all $x \in K_{n}$. Let $v$ be an increasing homeomorphism mapping $[\beta(k), \alpha(k+1)]$ onto $[0,1]$. Define $\varphi_{n}: A_{n} \rightarrow C_{b}\left(T_{\alpha(n)}, B\right)$ by

$$
\varphi_{n}^{t}(a)=\left\{\begin{array}{lll}
\eta_{k}^{u(t)}\left(p_{k n}(a)\right) & \text { if } \quad \alpha(k) \leq t \leq \beta(k) \\
H_{k}^{v(t), \alpha(k+1)}\left(p_{k n}(a)\right) & \text { if } \quad \beta(k) \leq t \leq \alpha(k+1)
\end{array}\right.
$$

This is well defined by (27). Using (25), (26) and the choice of $u(t)$ it easily seen that $\lim _{t \rightarrow \infty}\left\|\varphi_{n}^{t}(x)-\varphi_{t} p_{n}(x)\right\|=0$ for all $x \in K_{n}$.

Since $\hat{\varphi}_{k} p_{k n}=\hat{\varphi}_{n}$ for all $k \geq n$ and $\overline{\cup p_{n}\left(K_{n}\right)}=A$ it follows that $\hat{\varphi}_{n}=\hat{\varphi} \circ p_{n}$ for all $n$.

Corollary 3.15 Let $\underline{A}=\left(A_{n}, p_{n+1 n}\right), \underline{B}=\left(B_{n}, q_{n+1 n}\right)$ be inductive systems

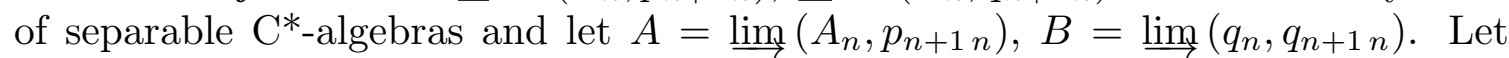
$\varphi_{t}: A \rightarrow B$ be an asymptotic morphism. If $\left(A_{n}, p_{n+1 n}\right)$ is semiprojective then there is a strong map of systems $(f, \underline{h}): \underline{A} \rightarrow \underline{B}$ and an increasing map $\alpha: \mathbb{N} \rightarrow \mathbb{N}$ such that $L_{0}(\underline{f}, \underline{h})$ is equivalent to $\varphi_{\tilde{\alpha}(t)}$.

Proof Let $\varphi_{n}$ be as in the conclusion of Proposition 3.14. Define a strong map of systems $(\underline{f}, \underline{h})$

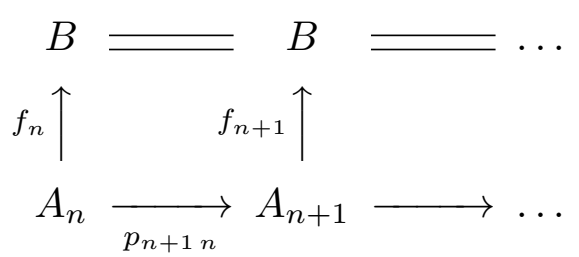

by setting $f_{n}: A_{n} \rightarrow B, f_{n}=\varphi_{n}^{\alpha(n)}$ and $h_{n}: A_{n} \rightarrow B[0,1], h_{n}^{\tau}=\varphi_{n}^{(1-\tau) \alpha(k)+\tau \alpha(k+1)}$. A simple calculation shows that $L_{0}(\underline{f}, \underline{h})$ is equivalent to the asymptotic morphism $\varphi_{\tilde{\alpha}(t)}$.

Since the inductive system $\underline{A}$ is semiprojective by using Theorem 3.3 in $[\mathrm{B}],(\underline{f}, \underline{h})$ lifts to a map of systems $\underline{A} \rightarrow \underline{B}$. We omit the details.

3.16 Blackadar showed in $[B]$ that every separable $C^{*}$-algebra is the inductive limit of some semiprojective inductive system. We recall here his construction. Let $A$ be a separable $\mathrm{C}^{*}$-algebra and let $\mathcal{G}$ be a dense selfadjoint countable subring of $A$ which is an algebra over the rational numbers. Let $\mathcal{R}$ denote the set of all *-algebraic relations on $\mathcal{G}$ plus the scalar multiple relations between elements of $\mathcal{G}$ plus a relation $\|x\| \leq\|x\|_{A}$ for each $x \in \mathcal{G}$. Then the $\mathrm{C}^{*}$-algebra $A$ is isomorphic to the universal $\mathrm{C}^{*}$-algebra on the set $\mathcal{G}$ of generators and the set of relations $\mathcal{R}$. Write $\mathcal{G}=\left\{x_{1}, x_{2}, \ldots\right\}$ and

$$
\mathcal{R}=\left\{\left(\left\|p_{1}(.)\right\| \leq c_{1}\right),\left(\left\|p_{2}(.)\right\| \leq c_{2}\right), \ldots\right\} .
$$

Let $\mathcal{G}_{n}=\left\{x_{1}, \ldots x_{n}\right\}$ and

$$
\mathcal{R}_{n}=\left\{\left(\left\|\tilde{p}_{j}(.)\right\| \leq c_{j}+1 / n\right),\left(\left\|x_{j}\right\| \leq\left\|x_{j}\right\|_{A}+1 / n\right): 1 \leq j \leq n\right\}
$$

where $\tilde{p}_{j}=p_{j}$ if $p_{j}$ involves only $x_{1}, \ldots x_{n}$ and $\tilde{p}_{j}$ is void otherwise. Let $A_{n}$ be the universal $\mathrm{C}^{*}$-algebra on the set $\mathcal{G}_{n}$ of generators and the set of relations $\mathcal{R}_{n}$. There is a natural map $p_{n+1}: A_{n} \rightarrow A_{n+1}$ induced by the inclusion $\mathcal{G}_{n} \subset \mathcal{G}_{n+1}$. This is 
well defined since the relations in $\mathcal{R}_{n+1}$ include stronger forms of all the relations in $\mathcal{R}_{n}$. Finally one checks that $p_{n+1 n}$ is semiprojective and $A=\underline{\varliminf}\left(A_{n}, p_{n+1 n}\right)$.

In this circle of ideas we have the following easy Lemma whose proof is omitted.

Lemma 3.17 Let $\left(A_{n}, p_{n+1 n}\right)$ be as in 3.16. Then there is $\delta_{n}>0$ such that for any $\mathrm{C}^{*}$-algebra $B$ and any two $*$-homomorphisms $\varphi, \psi: A_{n+1} \rightarrow B$ satisfying $\|\varphi(x)-\psi(x)\| \leq \delta_{n}$ for all $x \in \mathcal{G}_{n+1}$, the formula

$$
h_{n}^{s}\left(x_{j}\right)=(1-s) \varphi\left(p_{n+1 n}\left(x_{j}\right)\right)+s \psi\left(p_{n+1 n}\left(x_{j}\right)\right)
$$

$1 \leq j \leq n$, extends to a well defined homotopy of $*$-homomorphisms $h_{n}: A_{n} \rightarrow$ $B[0,1]$ from $\varphi \circ p_{n+1 n}$ to $\psi \circ p_{n+1 n}$.

Proposition 3.18 Let $(\underline{f}, \underline{h}),\left(\underline{f}^{\prime}, \underline{h}^{\prime}\right): \underline{A} \rightarrow \underline{B}$ be strong maps of systems. Supose that $\underline{A}$ is semiprojective. If $L_{0}(\underline{f}, \underline{h})$ is homotopic to $L_{0}\left(\underline{f}^{\prime}, \underline{h}^{\prime}\right)$ then $(\underline{f}, \underline{h})$ is homotopic to $\left(f^{\prime}, \underline{h}^{\prime}\right)$. Therefore

$$
L:[[\underline{A}, \underline{B}]] \rightarrow[[\underline{\underline{i m}} \underline{A}, \underline{\lim } \underline{B}]]
$$

is injective.

Proof Let $A=\underline{\varliminf} \underline{A}$ and $B=\underline{\lim } \underline{B}$. We begin by proving Proposition 3.18 under two additional assumptions:

(a) $L_{0}(\underline{f}, \underline{h})$ is equivalent to $L_{0}\left(\underline{f}^{\prime}, \underline{h}^{\prime}\right)$.

(b) $\underline{A}=\left(A_{n}, p_{n+1 n}\right)$ is as in 3.16 .

We may suppose that $(\underline{f}, \underline{h})$ and $\left(\underline{f}^{\prime}, \underline{h}^{\prime}\right)$ have the same indexing map equal to the identity of $\mathbb{N}$. Recalling the construction of $L_{0}$ given in 2.2 it is obvious that if $L_{0}(\underline{f}, \underline{h})$ is equivalent to $L_{0}\left(\underline{f}^{\prime}, \underline{h}^{\prime}\right)$ then

$$
\lim _{t \rightarrow \infty}\left\|q_{f(k+1)}\left(h_{k}^{t-k}-h_{k}^{\prime t-k}\right) p_{k n}(x)\right\|=0
$$

for all $x \in A_{n}$, where $k$ denotes the integer part of $t$. Since each $A_{n}$ is finitely generated, after replacing $(\underline{f}, \underline{h})$ by $\underline{i}_{\beta} \circ\left((\underline{f}, \underline{h}) \circ \underline{i}_{\alpha}\right)$ and $\left(\underline{f}^{\prime}, \underline{h}^{\prime}\right)$ by $\underline{i}_{\beta} \circ\left(\left(\underline{f}^{\prime}, \underline{h}^{\prime}\right) \circ \underline{i}_{\alpha}\right)$ we may assume that

$$
\lim _{t \rightarrow \infty}\left\|\left(h_{k}^{t-k}-h_{k}^{\prime t-k}\right) p_{k n}(x)\right\|=0
$$

for all $x \in A_{n}$, where $k$ denotes the integer part of $t$ and moreover

$$
\left\|h_{k}^{t-k} p_{k n}(x)-h_{k}^{\prime t-k} p_{k n}(x)\right\| \leq \delta_{n}, \quad\left\|f_{n}(x)-f_{n}^{\prime}(x)\right\| \leq \delta_{n}
$$

for all $x \in \mathcal{G}_{n}$ and $k \geq n$, where $\mathcal{G}_{n}$ and $\delta_{n}$ are as in Lemma 3.17.

Let $\alpha: \mathbb{N} \rightarrow \mathbb{N}, \alpha(n)=n+1$ and consider the strong maps of systems $\left(\underline{f}^{\alpha}, \underline{h}^{\alpha}\right)$, $\left(\underline{f}^{\prime \alpha}, \underline{h}^{\prime \alpha}\right): \underline{A} \rightarrow \underline{B}($ see $(2.5))$. We have

$$
\begin{aligned}
f_{n}^{\alpha} & =f_{n+1} p_{n+1 n}: A_{n} \rightarrow B_{n+1} \\
h_{n}^{\alpha} & =h_{n} p_{n+1 n}: A_{n} \rightarrow B_{n+2}[0,1] \\
f_{n}^{\prime \alpha} & =f_{n+1}^{\prime} p_{n+1 n}: A_{n} \rightarrow B_{n+1} \\
h_{n}^{\prime \alpha} & =h_{n}^{\prime} p_{n+1 n}: A_{n} \rightarrow B_{n+2}[0,1]
\end{aligned}
$$


As a consequence of $(29)\left\|h_{n+1}(x)-h_{n+1}^{\prime}(x)\right\| \leq \delta_{n+1}$ for all $x \in \mathcal{G}_{n+1}$. Thus by Lemma 3.17 the formula

$$
\mu_{n}^{s, \tau}\left(x_{j}\right)=(1-s) h_{n+1}^{\tau}\left(p_{n+1 n}\left(x_{j}\right)\right)+s h_{n+1}^{\prime \tau}\left(p_{n+1 n}\left(x_{j}\right)\right)
$$

with $x_{j} \in \mathcal{G}_{n}$ extends to a well defined $*$-homomorphism $\mu: A_{n} \rightarrow B_{n+2}[0,1] \times[0,1]$. Similarly there is a unique $*$-homomorphism $\nu_{n}: A_{n} \rightarrow B_{n+1}[0,1]$ such that $\nu_{n}^{s}\left(x_{j}\right)=(1-s) f_{n+1} p_{n+1 n}\left(x_{j}\right)+s f_{n+1}^{\prime} p_{n+1}\left(x_{j}\right)$ for all $x_{j} \in \mathcal{G}_{n}$. In this way we obtain a strong map of systems $\left(\nu_{n}, \mu_{n}\right): \underline{A} \rightarrow \underline{B}$ which is a homotopy between $\left(\underline{f}^{\alpha}, \underline{h}^{\alpha}\right)$ and $\left(\underline{f}^{\prime \alpha}, \underline{h}^{\prime \alpha}\right)$.

We shall remove now assumption $(a)$. Let $\Phi_{t}: A \rightarrow B[0,1]$ be a homotopy of asymptotic morphisms such that $\Phi_{t}^{0}=L_{0}(\underline{f}, \underline{h})$ and $\Phi_{t}^{1}=L_{0}\left(\underline{f}^{\prime}, \underline{h}^{\prime}\right)$.

By Corollary 3.15 there is a strong map of systems $(\underline{\nu}, \underline{\mu}): \underline{A} \rightarrow \underline{B}$ such that $L_{0}(\underline{\nu}, \underline{\mu})$ is equivalent to $\Phi_{\tilde{\alpha}(t)}$ for some increasing map $\alpha: \mathbb{N} \rightarrow \mathbb{N}$. Lemma 2.6 shows that after replacing $(\underline{f}, \underline{h})$ by $\left(\underline{f}^{\alpha}, \underline{h}^{\alpha}\right)$ and $\left(\underline{f}^{\prime}, \underline{h}^{\prime}\right)$ by $\left(\underline{f}^{\prime \alpha}, \underline{h}^{\prime \alpha}\right)$ we may assume that $L_{0}(\underline{\nu}, \mu)$ is equivalent to $\Phi_{t}$. Then as in the proof of Lemma 2.3 we have that $L_{0}\left(\underline{e}_{0}(\underline{\nu}, \underline{\mu})\right)$ is equivalent to $\Phi_{t}^{0}$ hence equivalent to $L_{0}(\underline{f}, \underline{h})$. Similarly $L_{0}\left(\underline{e}_{1}(\underline{\nu}, \underline{\mu})\right)$ is equivalent to $L_{0}\left(\underline{f}^{\prime}, \underline{h}^{\prime}\right)$. By the first part of the proof it follows that $(\underline{f}, \underline{h})$ is homotopic to $\left(\underline{e}_{0}(\underline{\nu}, \underline{\mu})\right)$ and $\left(\underline{f}^{\prime}, \underline{h}^{\prime}\right)$ is homotopic to $\left(\underline{e}_{1}(\underline{\nu}, \underline{\mu})\right)$. Since homotopy of strong maps of systems is an equivalence relation we are done. To complete the proof we are going now to remove assumption $(b)$. Let $\underline{A}^{\prime}$ be any semiprojective inductive system with $\underline{\lim } \underline{A}^{\prime}=A$. By 3.3 this implies that $\underline{A}^{\prime}$ is isomorphic to $\underline{A}$ in the strong shape category $\mathcal{S}$. From what we have proven so far we know that $L$ induces an injective map

$$
L:[[\underline{A}, \underline{B}]] \rightarrow[[A, B]] .
$$

Since $L$ is a functor and $\underline{A}$ is isomorphic to $\underline{A}^{\prime}$ in $\mathcal{S}$ it follows that

$$
L:\left[\left[\underline{A}^{\prime}, \underline{B}\right]\right] \rightarrow[[A, B]]
$$

is injective too.

\section{REFERENCES}

[B] B. Blackadar, Shape theory for $C^{*}$-algebras, Math. Scand. 56 (1985), 249-275.

[Ca] F. Cathey, Strong Shape Theory, Thesis, University of Washington, 1979.

[C] A. Connes, Non-commutative Geometry, the English edition (in preparation).

[CH] A. Connes and N. Higson, Deformations, morphismes asymptotiques et K-theorie bivariante, C.R. Acad. Sci. Paris 313 (1990).

[CH1] A. Connes and N. Higson (in preparation).

[CK] A. Connes and J. Kaminker (in preparation).

[Cu] J. Cuntz, K-theory and $C^{*}$-algebras, Lectures Notes in Math., vol. 1946, Springer-Verlag, Berlin and New York, 1984, pp. 55-79.

[D] M. Dadarlat, A note on asymptotic morphisms, preprint 1992.

[D1] M. Dadarlat, Homotopy after tensorization by UHF algebras, to appear in J. of K-Theory.

[D2] M. Dadarlat, Some examples in homotopy theory of stable $C^{*}$-algebras, preprint 1991.

[D3] M. Dadarlat, On the asymptotic homotopy type of inductive limit $C^{*}$-algebras, to appear in Math. Annalen.

[DN] M. Dadarlat and A. Nemethi, Shape theory and connective K-theory, J. of Operator theory 23 (1990), 207-291.

[DL] M. Dadarlat and T. A. Loring, K-homology, asymptotic representations and unsuspended E-theory, preprint, 1992. 
[EH] D. A. Edwards and H. M. Hastings, Čech and Steenrod homotopy theories with applications to geometric topology, Lecture Notes in Math. no. 542, Spinger-Verlag, Berlin and New York, 1976.

[EK1] E. G. Effros and J. Kaminker, Homotopy continuity and shape theory for $C^{*}$-algebras, Geometric methods in operator algebras, U.S.-Japan seminar at Kyoto 1983, Pitman 1985.

[EK2] E. G. Effros and J. Kaminker, Some homotopy and shape computations, Group Representations, Ergodic Theory, Operator Algebras and Mathematical Physics (C. C. Moore, ed.), MSRI series no. 6 1986, Springer-Verlag.

[E] G. Elliott, Are amenable $C^{*}$-algebras classifiable?, Representation Theory of Groups and Algebras (J.Adams, R. Herb, S. Kudla, J.-S. Li, R. Lipsman, and J. Rosenberg, eds.), Contemp. Math. no. 145, Amer. Math. Soc., 1993, pp. 423-427.

$[\mathrm{H}] \quad$ N. Higson, Categories of fractions and excision in K-theory, J. of Pure and Appl. Algebra 65 (1990), 119-138.

[K] G. G. Kasparov, The operator K-functor and extensions of $C^{*}$-algebras, Math. URSS Izv. 16 (1981), 513-572.

[L] T. Ju. Lisica, On the exactness of the spectral homotopy group sequence in shape theory, Soviet Math. Dokl. 18 (1977), 1118-1190.

[Lo] T. A. Loring, C*-algebras generated by stable relations, J. Funct. Anal. 112 (1993), 159-201.

[MS] S. Mardešić and J. Segal, Shape Theory, North-Holland, Amsterdam, 1982.

[Q] J. B. Quigley, An exact sequence from the $n$th to the (n-1) st fundamental group, Fund. Math. 77 (1973), 195-210.

[RS] J. Rosenberg and C. Schochet, The Künneth theorem and the universal coefficient theorem for Kasparov's generalized functor, Duke Math. J. 55 (1987), 431-474.

Department of Mathematics, Purdue University, West Lafayette, IN 47907 USA 